\title{
Seasonal dynamics and mortality rates of Calanus helgolandicus over two years at a station in the English Channel
}

\author{
A. G. Hirst ${ }^{1, *}$, D. Bonnet ${ }^{2,3}$, R. P. Harris ${ }^{2}$ \\ ${ }^{1}$ British Antarctic Survey, Natural Environment Research Council, High Cross, Madingley Road, Cambridge CB3 0ET, UK \\ ${ }^{2}$ Plymouth Marine Laboratory, Prospect Place, Plymouth PL1 3DH, UK \\ ${ }^{3}$ Present address: Laboratoire Ecosystèmes Lagunaires, Place Eugène Bataillon, CC093, 34095 Montpellier Cedex 05, France
}

\begin{abstract}
The stage-specific abundance and egg production rates of Calanus helgolandicus were determined on a near-weekly basis over $2 \mathrm{yr}$ at a $50 \mathrm{~m}$ deep station in the SW English Channel (Stn L4). Mortality rates were derived using a vertical life-table approach across eggs, nauplii and also the $\mathrm{CV}$-adult stage pair. The results demonstrate strong seasonal patterns in the mortality rates of egg and nauplii and the CV-adult stage pair, but with different relative rates and somewhat different seasonalities. Mortality was highest in the egg and egg-NI stages, averaging 6.1 and $\sim 1.5 \mathrm{~d}^{-1}$, respectively, with the percentage surviving through the egg-NI stage pair often $<<10 \%$. Although the instantaneous removal rate of eggs was significantly related to adult abundance $\left(\mathrm{p}<0.001, \mathrm{r}^{2}=\right.$ 0.221), densities of adult $C$. helgolandicus seemed too low to account for these rates. Examination of the relationship between $\mathrm{CV}$-adult female mortality and the abundance of the dominant invertebrate predators revealed statistically significant relationships $\left(\mathrm{p}<0.001 \mathrm{r}^{2}=0.276\right.$ for chaetognaths; $\mathrm{p}<$ $0.001 \mathrm{r}^{2}=0.125$ for siphonophores); however, the variability explained by temperature was much higher $\left(\mathrm{p}<0.001 \mathrm{r}^{2}=0.652\right)$. The egg-NI and NI-NII stage pairs also showed a highly statistically significant positive relationship between mortality and temperature. For the first time we compared mortality rates for egg-NI using 2 vertical methods - one using measurements of egg and NI abundance (Method A) the other using egg production rates and NI abundance (Method B) — and found the two to be similar, although Method B gave higher values. Finally, as many mortality equations do not consider the bias resulting from the presence of eggs incapable of hatching in the field, we derived and applied new equations for mortality of eggs and egg-NI (Method A) that incorporated egg hatching success. At low hatching success or low mortality rates, this correction can alter estimates of mortality rates significantly.
\end{abstract}

KEY WORDS: Copepod $\cdot$ Mortality $\cdot$ Fecundity $\cdot$ Seasonality $\cdot$ Stage-specific

Resale or republication not permitted without written consent of the publisher

\section{INTRODUCTION}

While much effort has been expended in understanding the positive terms in the mass balance of copepod population abundance and biomass, i.e. growth and fecundity (see syntheses of Hirst \& Bunker [2003] and Bunker \& Hirst [2004]), we have a much more restricted understanding of the loss terms, i.e. mortality; yet mortality can be more important than growth in controlling abundance and biomass (Uye et al. 1992). Many advances have been made in the study of copepod mortality over the last decade, with more measurements in a greater range of environments and circumstances (Ohman \& Hirche 2001, Eiane et al. 2002, Ohman et al. 2004). However, there are still relatively few measurements in relation to other demographic parameters (see Hirst \& Kiørboe 2002), and we need to greatly improve our understanding of the seasonality of mortality rates and the agents that are critical in their control. 
In this study, we set out to examine the rates of mortality of Calanus helgolandicus, a copepod species common in the shelf regions of the Atlantic coast of Europe and across a large section of the North Atlantic (Continuous Plankton Recorder Survey Team 2004). We examined specifically its abundance, fecundity and mortality rates across egg-nauplii stages and CV-adult stage pairs over 2 annual cycles, from March 2002 to March 2004, at Stn L4, a 50 m deep station in the English Channel off Plymouth, UK. This station is the location of a long-time series of plankton observations (Irigoien \& Harris 2003). Previous studies have investigated the dietary controls on fecundity of $C$. helgolandicus (Pond et al. 1996); however, as yet there has been no examination of mortality at Stn L4. Temperature at Stn L4 fluctuates from 8.5 to $18^{\circ} \mathrm{C}$ over the course of a year, and there is little evidence of any significant inter-annual variability (see www.pml.ac.uk/ L4/). The water column is generally well mixed, being only weakly stratified from spring to autumn (Holligan \& Harbour 1977, Pingree et al. 1978, Holligan et al. 1984). Like most marine environments, Stn L4 is affected by advection; flows are largely determined by wind and tide, with the main current velocities ranging between 3 and $5 \mathrm{~cm} \mathrm{~s}^{-1}$, representing a distance of transport between 2.6 and $4.3 \mathrm{~km} \mathrm{~d}^{-1}$. Horizontal approaches to determining mortality can only be used where advective loss or gain of animals is extremely restricted and relatively unimportant in comparison to mortality. As a consequence of the hydrography, we therefore limited our methods to vertical approaches when determining mortality (Mullin \& Brook 1970, Aksnes \& Ohman 1996).

\section{MATERIALS AND METHODS}

Field collection. Net samples were collected on a near-weekly basis from March 2002 to March 2004 at Stn L4 in the English Channel. Two WP2 nets, 50 and $200 \mu \mathrm{m}$ mesh, were hauled vertically from the sea floor ( $\sim 50 \mathrm{~m}$ deep) to the surface. Upon return to the laboratory, samples were preserved in $5 \%$ buffered formaldehyde for stage-analysis. Temperature and chlorophyll a were determined from surface water collections. Chlorophyll a was estimated from 3 replicates (100 ml each) of seawater filtered onto GF/F filters. Pigments were extracted in $10 \mathrm{ml}$ of $90 \%$ acetone over $24 \mathrm{~h}$ in the dark at $4^{\circ} \mathrm{C}$. Fluorescence was measured with a Turner fluorometer.

Stage abundance. Prior to enumerating stages, fine mesh samples were subsampled with a Stempel pipette, so that the aliquot counted contained around 100 individuals. All stages, eggs, nauplii and copepodites were enumerated in these fine-mesh samples.
The entire $200 \mu \mathrm{m}$ net sample was counted for adult males and females. Subsequently it was subsampled and around 100 Stages CI-CV were counted. In deriving mortality we used the egg and nauplii abundance values from the $50 \mu \mathrm{m}$ mesh net, while for copepodites and adults we used the $200 \mu \mathrm{m}$ mesh net values.

Egg production. Slow, gentle, short-duration tows were made using a $500 \mu \mathrm{m}$ net. The contents of the cod end were emptied into $10 \mathrm{l}$ buckets of surface seawater, and kept in cool boxes until return to the laboratory (typically within $2 \mathrm{~h}$ ). In the laboratory, adult female Calanus helgolandicus were picked out and groups of 5 individuals were incubated in 21 beakers each with Plexiglas inserts with $300 \mu \mathrm{m}$ mesh false bottoms to separate eggs from females and reduce any cannibalism. A total of 5 container replicates were set up (i.e. a total of 25 individuals). Incubations were for $24 \mathrm{~h}$ at $15^{\circ} \mathrm{C}$ with a $12: 12 \mathrm{~h}$ dark:light cycle. At the end of the incubation females were recounted and their condition checked. Eggs were removed using a $50 \mu \mathrm{m}$ mesh and counted. Egg production $\left(\mathrm{d}^{-1}\right)$ was calculated from the total number of eggs produced divided by the number of females alive at the end of the incubation. Later in the course of this investigation egg hatching experiments were added. The methodology we followed to determine egg hatching success was that at the end of the egg production experiment, 24 eggs were isolated and placed individually in $2 \mathrm{ml}$ wells of a multiplate, and held at $15^{\circ} \mathrm{C}$. Egg hatching success was followed at $24 \mathrm{~h}$ intervals for a full $5 \mathrm{~d}$. Any eggs that had not hatched by the end of this period were deemed to be non-hatchers and the percentage egg hatching was derived accordingly.

Stage duration. Accurate independent estimates of development times are necessary in order to determine mortality rates using vertical methods. There is a general lack of stage-specific development times for Calanus helgolandicus in the literature. Unfortunately the extensive data of Thompson (1982) is likely to comprise a mix of C. helgolandicus and C. finmarchicus. Stage duration experiments for nauplii and CV copepodites were therefore conducted on $C$. helgolandicus at 3 temperatures $\left(9,12\right.$ and $\left.15^{\circ} \mathrm{C}\right)$; the methods used and rates measured are to be published elsewhere (D. Bonnet et al. unpubl.). These data were used in combination with rates compiled from other studies in order to describe development time $(D)$ as a function of temperature $(T)$. As most data were available for eggs we first solved for this stage using a Bêlehràdek function of the form:

$$
D=a(T-\alpha)^{-b}
$$

We set $b$ at 2.05 before solving using curve-fitting software (CurveExpert 1.38, Microsoft). This $b$ value is commonly applied throughout the literature and 
regarded as representative for copepods (e.g. see Corkett et al. 1986). We then fitted this equation to the egg stage, for which we had most data, and this produced a value for $\alpha$ of -8.974 . Subsequently, we fitted Bêlehràdek functions to the other stages, applying a $b$ value of 2.05 and $\alpha$ of -8.974 ; hence we solved for $a$ using the data available. The resulting equations and sources of data are detailed in Table 1.

Mortality estimation. Application of the population surface method (PSM) is not appropriate at Stn L4 because of the need to link stage abundance through time (i.e. horizontally) (Wood 1994), whereas Stn L4 experiences considerable advection. As might be expected, attempts to use a PSM approach on the data proved unsuccessful (K. Eiane pers. comm.). We therefore used a vertical life-table (VLT) approach to solve rates of mortality in paired stages. Advection only becomes a problem when using a VLT approach if transport processes do not equally influence stage pairs over the period corresponding to the total duration of the 2 stages. As stages can have different vertical distribution, and advection can also change with depth, we cannot discount that this may have an impact; however, averaging across sampling events should reduce any bias. The other main assumption behind the VLT approach is that there should not be a trend in recruitment to a stage over the duration of the combination of stages for which mortality is being determined (Aksnes et al. 1997). There was little evidence for the existence of strong cohorts during our study (see Figs. 1 \& 2), although there were temporal trends in the rate of recruitment, i.e. total egg production rate (see Fig. 4b). To examine whether changes in recruitment rate (as total egg production) would have an important impact upon the mortality rates for eggs and egg-NI we split all total egg production data into the periods over which consistent trends in this term

Table 1. Calanus helgolandicus. Bêlehràdek functions derived and applied in this study to determine stage-specific development times $(D, \mathrm{~h})$. Egg hatching times fitted the equation $D=a(T-\alpha)^{-2.05}$ and $\alpha$ was determined as -8.974 ; subsequently this value was applied across all later stages. A curve-fitting program (Curve Expert 1.38, Microsoft) was used in all cases. All C. helgolandicus were collected in Channel waters off Plymouth, except those in study of K. Cook et al. (pers. comm.) which came from Stonehaven (northern North Sea)

\begin{tabular}{|lccl|}
\hline Stage & \multicolumn{1}{c|}{$\alpha$} & $\alpha$ & Source \\
\hline Egg & 17466 & -8.974 & $\begin{array}{l}\text { Corkett (1972), Rey et al. (2001), D. Bonnet et al. } \\
\text { (unpubl.), K. Cook et al. (pers. comm.) }\end{array}$ \\
NI & 10173 & As for eggs & K. Cook et al. (pers. comm.) \\
NII & 19044 & As for eggs & D. Bonnet et al. (unpubl.), K. Cook et al. (pers. comm.) \\
NIII & 49831 & As for eggs & D. Bonnet et al. (unpubl.) \\
NIV & 34790 & As for eggs & Rey et al. (2001), D. Bonnet et al. (unpubl.) \\
NV & 34080 & As for eggs & Rey et al. (2001), D. Bonnet et al. (unpubl.) \\
NVI & 70069 & As for eggs & Rey et al. (2001), D. Bonnet et al. (unpubl.) \\
CV & 90266 & As for eggs & D. Bonnet et al. (unpubl.) \\
\hline
\end{tabular}

were observed (i.e. 19 March 2002 to 17 February 2003; 6 January 2003 to 6 May 2003; 3 March 2003 to 10 December 2003; 15 December 2003 to 29 March 2004). The slope of $\log _{\mathrm{e}}$ total egg production versus time (d) was then derived using linear regression for each of these 4 periods; the slopes of these relationships give an indication as to whether changes in recruitment are likely to cause serious errors in the mortality estimates. The slopes for Periods 1 to 4 were $-0.01,0.05,-0.01$ and $0.03 \mathrm{~d}^{-1}$ respectively (see Fig. 4b). These trends were typically 1 and sometimes 2 orders of magnitude lower than the mortality rates of eggs, egg-NI and NI-NII; hence trends in total egg production should not have had a significant bias on mortality rates of the early stages using the VLT approach. To further reduce the impact of any trends in any stages, and to also reduce impacts of sampling variability, we did not rely upon single mortality estimates; instead, as advisable, we averaged across many data points to produce stage averages (methods described later in this subsection).

Sub-adult stage mortality rates were estimated by iteration using the equation of Mullin \& Brooks (1970):

$$
\frac{\exp ^{\beta D_{i}}-1}{1-\exp ^{-\beta D_{i+1}}}=\frac{N_{i}}{N_{i+1}}
$$

where $\beta$ is mortality across stage pair ( $i$ and $i+1), N$ is the abundance of each stage $\left(\mathrm{m}^{-3}\right)$ and $D$ is the stage duration (d). Stage durations were predicted from the equations in Table 1 together with ambient surface seawater temperatures (the water column is generally well mixed, with $\mathrm{a} \ll 2^{\circ} \mathrm{C}$ temperature difference from surface to bottom throughout almost the entire year: Pond et al. 1996).

For the $\mathrm{CV}$-adult pair, the equation for mortality (Aksnes \& Ohman 1996) is:

$$
\beta=\frac{\ln \left(\frac{N_{\mathrm{CV}}}{N_{\text {adult }}}+1\right)}{D_{\mathrm{CV}}}
$$

Mortality rates were derived for total $\mathrm{CV}$-adult, i.e. without regard to the sex of the adults, and also separately for $\mathrm{CV}$-adult female and CV-adult male. We had counts for each of the sexes in the adult stage and assumed an unbiased sex ratio of 1:1 in the CV stage. As there is no evidence of late copepodite stages of Calanus helgolandicus entering a non-moulting resting phase in winter at this station, we therefore applied the Bêlehràdek functions in Table 1 for CVs throughout the year. However, as development times in the field may be longer than those under 
high food abundance in the laboratory, we also estimated arithmetic average mortality rates across the entire sampling period assuming that the durations of the feeding stages (NIII-CV) were twice those predicted from the Bêlehràdek functions in Table 1. We chose this value because development times cannot be extended indefinitely, and in the work of Campbell et al. (2001) on Calanus finmarchicus, the difference in development times between low $\left(\sim 25 \mu \mathrm{g} \mathrm{C} \mathrm{l}^{-1}\right)$ and high $\left(\sim 350 \mu \mathrm{g} \mathrm{C} \mathrm{l}^{-1}\right)$ food concentrations for both nauplii and copepodites was around 2-fold.

From measurements of egg production rates, it is possible to derive egg-NI mortality using another approach (hereafter termed Method B, whereas application of Eq. [2] to egg-NI is termed Method A). Mortality is solved by iteration using the equation (modified from Aksnes et al. 1997 with respect to stages):

$$
\frac{\exp ^{-\beta_{\text {egg-NI }} D_{\text {egg }}\left(1-\exp ^{-\beta_{\text {egg-NI }} D_{\mathrm{NI}}}\right)}}{\beta_{\text {egg-NI }}}=\frac{N_{\mathrm{I}}}{F \times N_{\text {adult_female }}}
$$

where $\beta_{\text {egg-NI }}$ is mortality across egg-NI stage pair $\left(\mathrm{d}^{-1}\right), F$ is the fecundity rate (eggs female ${ }^{-1} \mathrm{~d}^{-1}$ ), $N_{\text {adult_female }}$ is the abundance of adult females $\mathrm{m}^{-3}$ (the product of $F$ and $N_{\text {adult_female }}$ is the total number of eggs produced $\left.\mathrm{m}^{-3} \mathrm{~d}^{-1}\right), N_{\mathrm{I}}$ is the abundance of NI $\left(\mathrm{m}^{-3}\right)$, $D_{\text {egg }}$ is the stage duration of eggs (d) and $D_{\mathrm{NI}}$ that of NI. This method differs from Method A in that it does not rely upon having an accurate estimate of egg abundance in the water column, but uses the input of eggs from egg production experiments combined with the field abundance of adult females. We were able to compare mortality results from Methods A and B (Eqs. 2 \& 4, respectively) for the first time.

We obtained rates of mortality for the egg stage alone $\left(\beta_{\text {egg }}\right.$ ) using the vertical approach of Peterson \& Kimmerer (1994), as modified and corrected by Hirst \& Kiørboe (2002):

$$
\frac{N_{\text {egg }} \beta_{\text {egg }}}{F \times N_{\text {CVI_female }}}=\left[1-\mathrm{e}^{\left(-\beta_{\text {egg }} D_{\text {egg }}\right)}\right]
$$

In this study we only present mortality rates for the eggs and nauplii stage, and the CV-adult stage pair. There were little data for development times of copepodite stages, and often negative mortality rates were returned, hence their exclusion. Mortality estimates from the vertical approach are best averaged across several individual values (Aksnes \& Ohman 1996). We present arithmetic averages for the entire $2 \mathrm{yr}$ sampling period. In the case of eggs and non-feeding nauplii, and CV-adult we also present temporal descriptions. To allow us to average values, as necessary in a VLT approach, whilst also allowing this temporal description, we used a Lowess smoothing fit (SigmaPlot 2001, Version 8.02). With the Lowess method we chose an f-value of 0.2 . This value defines the amount of smoothing, and corresponds to the fraction of data points used for each regression. It was set at this level to give a reasonable reliance upon many data points whilst still showing smaller-scale temporal variability (our observations suggest that higher f-values have a tendency to suppress clear seasonal patterns in mortality - see 'Results'). Negative mortality estimates at individual time points are not uncommon when using the vertical approach (Aksnes \& Ohman 1996), and we have not removed such values; indeed, removal is unadvisable as this would give a positive bias to the results. Later, when examining mortality rates as a function of predator abundance and temperature, Lowess-smoothed values for each of the sampling dates were used rather than relying upon nonaveraged data.

The proportion surviving $(S)$ in a stage pair $\mathrm{d}^{-1}$ was determined from Peterson \& Kimmerer's (1994) equation:

$$
S=\exp (-\beta)
$$

We adapted this equation so we could determine the proportion surviving through the Stage Pair $i$ and $i+1$ $(T)$ :

$$
T=S^{D_{i}+D_{i+1}}
$$

Calanus spp. egg production can have diel periodicity, but as we had no measurements of this we could not allow for it in our estimates of mortality. As our sampling was in daylight hours we accept that strong discontinuities in the egg production rate could potentially cause some error in our mortality estimates.

Abundance of predators. Abundance of the major predator groups at Stn L4-siphonopores (individual numbers not simply counting colonies), chaetognaths, hydromedusae, euphausiids and other copepod species-were also determined from the $200 \mu \mathrm{m}$ samples. These results allowed us to explore the causes of mortality on CV-adult female mortality. We performed both linear regression analysis to explore the relationship between mortality of $\mathrm{CV}$-adult female and predator abundance, and multiple linear regressions including all of the predators.

\section{RESULTS}

\section{Stage abundance}

There was clear seasonality in the abundance of stages over the course of the investigation (Figs. 1 \& 2). Highest abundance was observed in February to August across all stages. The oldest copepodite stages were most abundant in February to June. Eggs were 

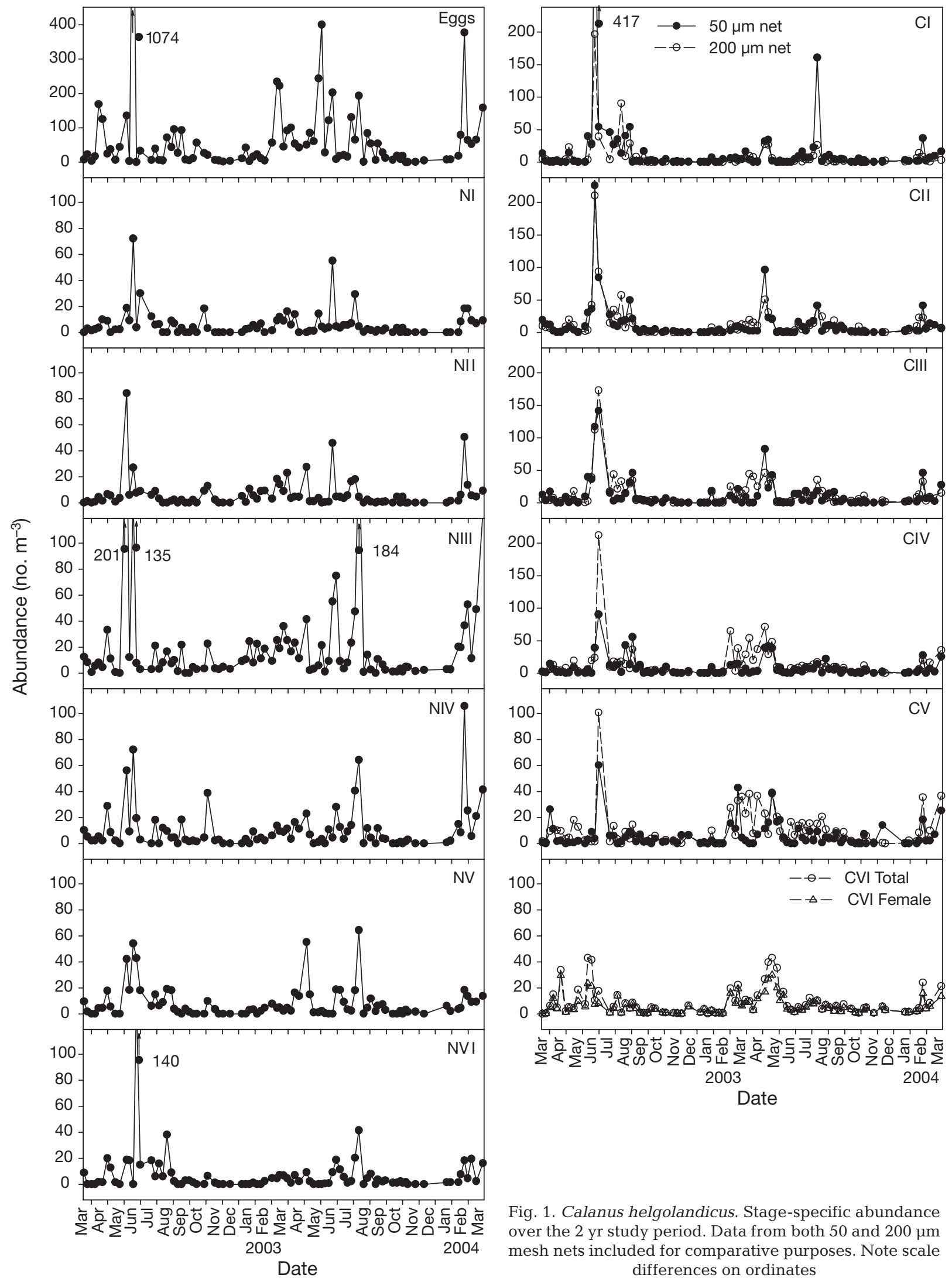

Fig. 1. Calanus helgolandicus. Stage-specific abundance over the 2 yr study period. Data from both 50 and $200 \mu \mathrm{m}$ mesh nets included for comparative purposes. Note scale differences on ordinates 


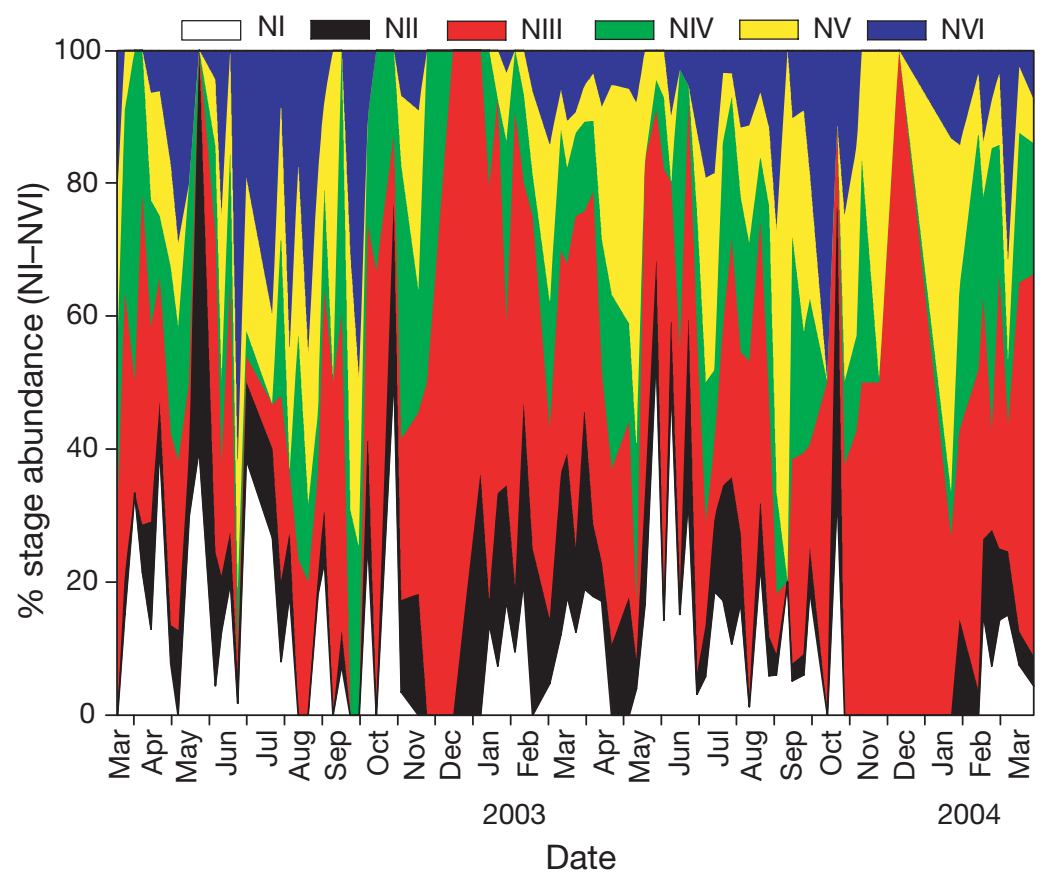

Fig. 2. Calanus helgolandicus. Percentage stage abundances of all nauplii across the 2 yr of sampling. All abundance values for $50 \mu \mathrm{m}$ nets
NVI is presented in Fig. 3. The durationcorrected abundances declined across eggs, NI and NII; however, NIII values were greater than NII when using laboratory ( food-saturated) durations (Fig. 3). NIII is the first feeding stage, and we suggest that its high abundance is strongly indicative of development time of this stage being food-limited in the field compared with the laboratory. After NIII there was a stage-on-stage decline in the duration-corrected abundance values across the $50 \mu \mathrm{m}$ net-sampled nauplii. For mortality estimation we used abundance from the fine $(50 \mu \mathrm{m})$ mesh net for enumeration of eggs and nauplii, while the coarse $(200 \mu \mathrm{m})$ mesh was used for $\mathrm{CV}$ and adults. We determined mortality using both development times predicted from the laboratory, and also assuming that field values were twice the laboratory times for in the feeding stages (NIII-CV).

\section{Egg production}

by far the most abundant stage on average, followed by NIII; this later stage had a longer stage duration than those stages which preceded it, hence its high abundance.

For each sampling date, stage-specific abundance values were divided by stage duration (at prevailing temperature). The average of these values for eggs to

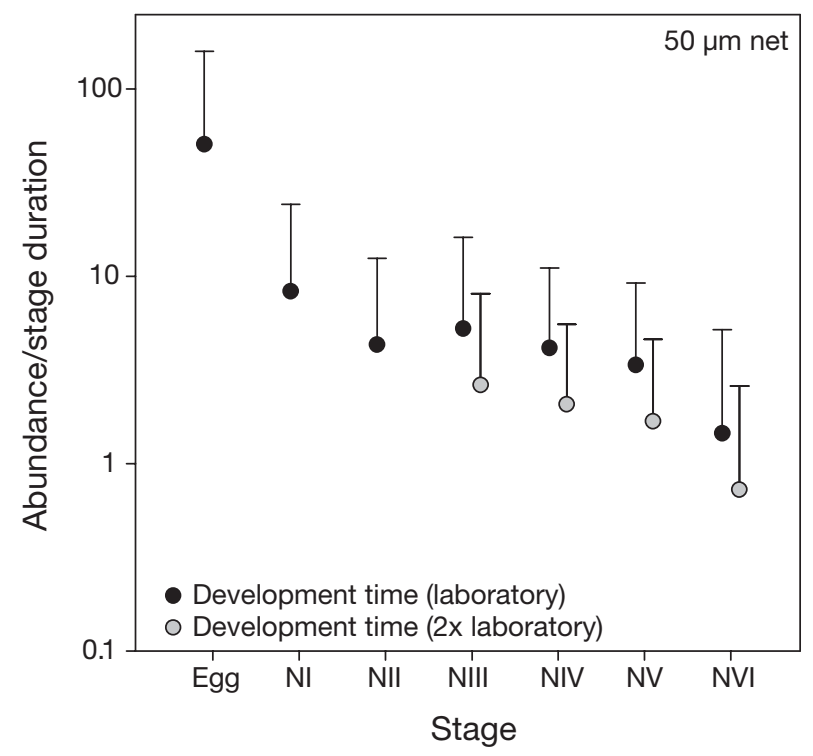

Fig. 3. Calanus helgolandicus. Mean (+95\% CI) stage-specific abundances divided by stage duration. Nauplii values presented for $50 \mu \mathrm{m}$ mesh net over the course of the study
There were clear temporal changes in rate of fecundity for both individuals and the population at Stn L4 (Fig. 4a,b). The highest rates for individuals were from April to June in 2002 and 2003, when they could exceed 40 to 50 eggs female ${ }^{-1} \mathrm{~d}^{-1}$ (Fig. 4a). Total rates of egg production by the Calanus helgolandicus population were highest in both years from April to June, reaching values $>600$ eggs $\mathrm{m}^{-3} \mathrm{~d}^{-1}$, while the entire range over the 2 yr was 0.3 to 820 eggs $\mathrm{m}^{-3} \mathrm{~d}^{-1}$ (Fig. 4b).

Egg hatching success was measured between June 2003 and March 2004. Over this period, success varied between 4 and 100\% (Fig. 4a). Generally, hatching success was high and varied only between $>70$ and $100 \%$ in the period June 2003 to August 2003. In September 2003 to late February 2004 hatching success was commonly $<70 \%$ and much more variable than in the earlier period.

\section{Mortality}

Arithmetic average rates of mortality over the $2 \mathrm{yr}$ of sampling are shown in Fig. 5. Highest mortality occurred in the egg stage at $6.08 \mathrm{~d}^{-1}$ (95\% confidence interval, CI, \pm 6.177 ). The next highest rates were across the egg-NI stage pair, with an average (Method A) of $1.30 \mathrm{~d}^{-1}(95 \% \mathrm{CI}, \pm 0.307)$, while the 

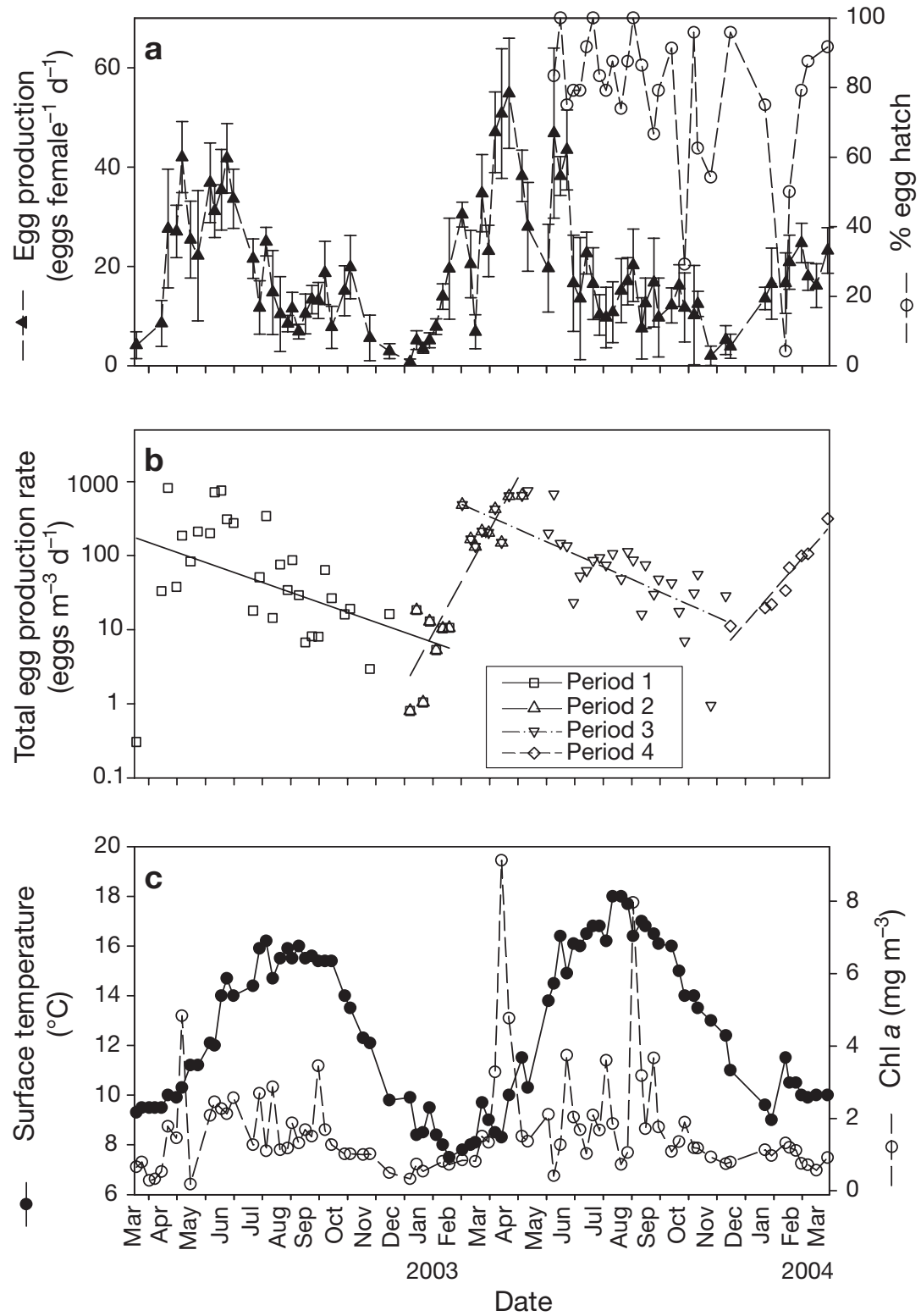

Fig. 4. Calanus helgolandicus. (a) Mean egg production at Stn L4. Error bars represent $\pm \mathrm{SD}$ of results from 5 replicates, together with percentage of eggs that successfully hatched during incubations. (b) Total egg production rates for the adult female population; data divided into 4 periods over which consistent trends in reproductive output were observed in order to test whether this might impact the mortality estimates using a vertical life-table approach. (c) Surface water temperature and surface chlorophyll a concentration

average for Method B was $1.72 \mathrm{~d}^{-1}(95 \% \mathrm{CI}, \pm 0.233)$; the 2 methods produced similar results, although the rates from Method B tended to be greater. Considering nauplii paired stages alone, these had the highest mortality values in the NI-NII stage pair at $0.95 \mathrm{~d}^{-1}$ $\left(95 \% \mathrm{CI}_{1} \pm 0.223\right)$. Values fell dramatically after this stage pair.

Fig. 5 shows mortality estimates for the various stages; those assuming that stage durations of feeding stages (NIII and beyond) were twice the prediction from the Bêlehràdek functions (Table 1) and are denoted by grey circles. The general pattern of rates across stages was similar to that for the unaltered rates, although absolute values were higher across NII-NIII, and lower in subsequent stages. The higher value across NII-NIII resulted from the longer duration of NIII but unaltered stage duration in NII (nonfeeding stage).

Mortality rates as a function of season for all non-feeding stages (egg-NII) are given in Fig. 6. To allow for averaging whilst still allowing a description of the seasonal pattern, Lowess smoothing was used. For eggs, we used 2 f-values ( $\mathrm{f}=$ 0.2 and 0.5 ) in determining Lowesssmoothed rates. We suggest that the higher f-value tends to lead to oversmoothing, and as a pragmatic approach used the lower f-value henceforth as this appeared to better capture the seasonal pattern indicated by the values themselves. Egg mortality rates peaked in May during both 2002 and 2003, while they were at their minimum throughout the October to February period (Fig. 6a). In 2003 there were 2 peaks in egg mortality, the first in May when Lowessaveraged rates rose to $8.2 \mathrm{~d}^{-1}$, and the second in August and September when they reached $6.6 \mathrm{~d}^{-1}$. Lowess-smoothed values for egg-NI never exceeded $4.1 \mathrm{~d}^{-1}$ in 2002 and showed a weaker seasonal pattern than in 2003.

The proportion (and percentage) surviving through the egg-NI stages are shown in Fig. 7. Usually less than $10 \%$ survived this stage pair, although there were periods in which survival did exceed this, for example through most of February to June, and also August and September. The mean proportion surviving (Method B) through the egg-NI over the $2 \mathrm{yr}$ of sampling was merely 0.09 (i.e. $9 \%$ ).

Mortality rates across the $\mathrm{CV}$-adult stage pair showed similar seasonal patterns in both sexes (Fig. 8), although the absolute rates differed markedly between males and females. Mortality rates for CV-adult male were almost always higher than for $\mathrm{CV}$-adult female, on average by a factor of 3.2 (Fig. 8). There was a small peak in mortality in March to April 2003 (and an indication of the tail of a peak in a similar period in 2002), and a second peak in July to September. 


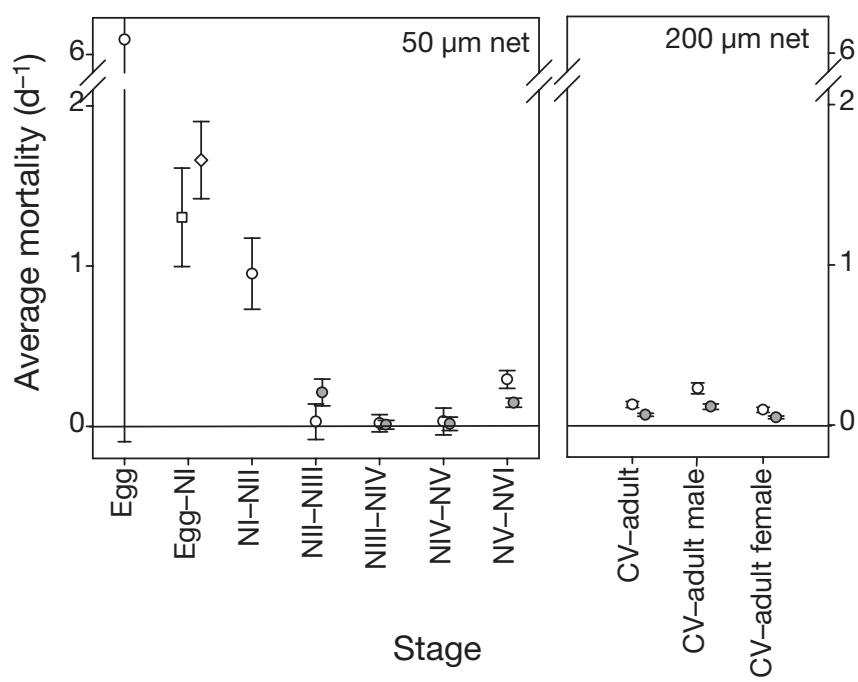

Fig. 5. Calanus helgolandicus. Arithmetic average ( $\pm 95 \% \mathrm{CI})$ mortality rates for eggs and nauplii and $\mathrm{C} 5$-adult stages, values averaged over the entire $2 \mathrm{yr}$ of sampling. Mortalities were obtained by assuming that stage durations were equal to those in the laboratory $(O)$ and that duration of feeding stages (NIII-CV) were twice laboratory rates (O). In the egg-NI stage pair, mortality was derived by both Method A () and Method B $(\diamond)$, see 'Materials and methods' for details

\section{Abundance of predators}

Abundances of predators over the 2 yr are presented in Fig. 9. Hydromedusae abundance was much lower than that of siphonophores or chaetognaths, reaching a maximum of 64 ind. $\mathrm{m}^{-3}$, although typically values were much lower than this. In both 2002 and 2003, chaetognaths showed an early abundance peak during June to July. In 2002, high numbers persisted throughout the rest of the year until late November. They also greatly outnumbered siphonophores in this year. There was a striking difference in 2003, however: after the initial peak of chaetognaths in June and July their abundance declined, and they never again reached those first peak levels. In contrast, siphophores became very numerous from late August 2003, and outnumbered chaetognaths. There was clear evidence of interannual differences in the abundance and relative dominance of pelagic predators at Stn L4 during this study.

Mortality rates of the $\mathrm{CV}$-adult female stage pair (Lowess-smoothed values used) were compared with the abundance of chaetognaths, hydromedusae and siphonophores. Regressions of mortality against both chaetognaths and siphonophores are shown in Fig. 10; both were statistically significant ( $p<0.001$ and 0.002 ), with predator abundance explaining 17 and $12 \%$ of the variability in mortality for chaetognaths and siphonophores, respectively. Plots for mortality versus hydromedusae abundance are not shown as the rela- tionship was not statistically significant $(p>0.05)$. Euphausiids were too rare to contribute significantly to the removal of Calanus helgolandicus and were therefore not examined further.

We compared both the egg mortality (Lowesssmoothed) and instantaneous egg removal rates (determined as the product of multiplying egg mortality rates by the egg density) with the abundance of Calanus helgolandicus adult females using linear regressions (Fig. 11a and b, respectively). Statistically significant relationships were observed $(p<0.001)$. The variability in instantaneous egg removal rates explained by the abundance of adult female $C$. helgolandicus was $22 \%$, while for egg mortality rates it was $19 \%$.

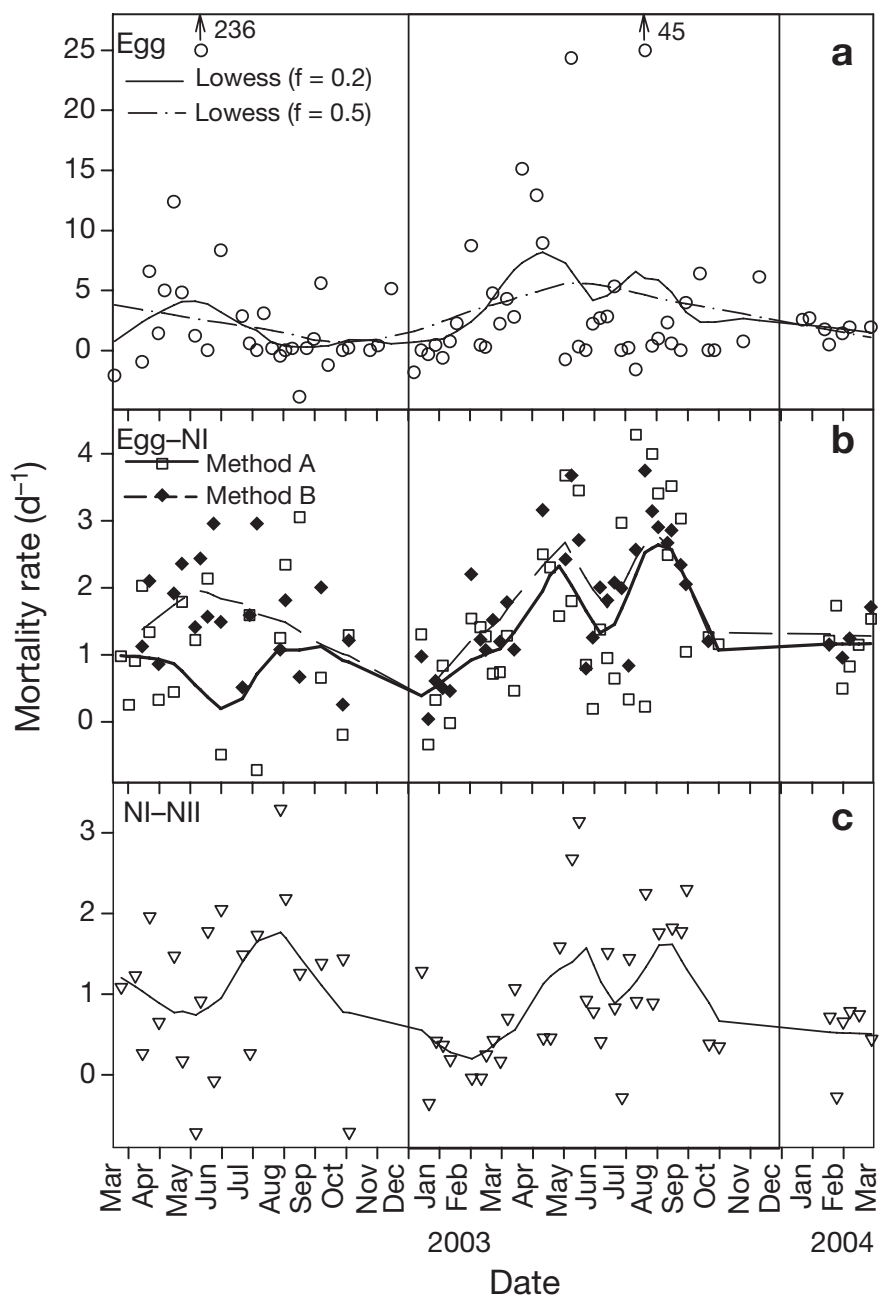

Fig. 6. Calanus helgolandicus. Mortality rates of non-feeding stages. (a) Eggs, (b) egg-NI and (c) NI-NII stage pairs derived from the vertical life-table approach using laboratory-determined stage durations. Each data point represents solution for an individual date. Lines indicate Lowess-fit through each data set with $\mathrm{f}=0.2$, except in (a), where Lowess-fit with $\mathrm{f}=0.5$ is also presented for comparison. Single high value of $236 \mathrm{~d}^{-1}$ for egg mortality was excluded when producing Lowess-fit for egg mortality 


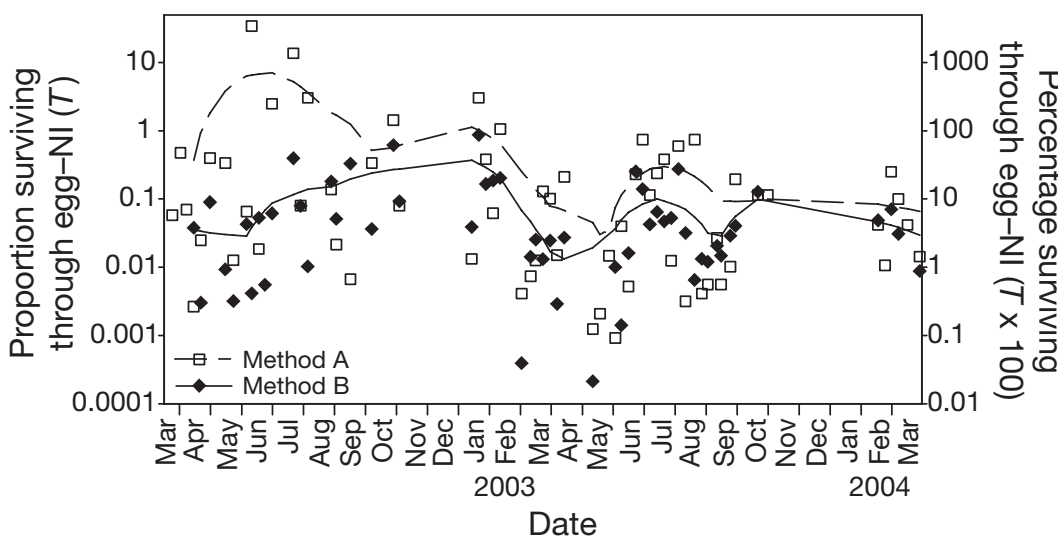

Fig. 7. Calanus helgolandicus. Proportion and Percentage surviving through egg-NI stages through the $2 \mathrm{yr}$ sampling period. Each data point represents solution for an individual date; lines indicate Lowess-fit through all data points

intercepts were often different from the predictions; egg-NI had higher rates than the model, as did NI-NII and CV-adult male, while CV-adult female had rates in close agreement in slope and intercept to that of Hirst \& Kiørboe (2002).

\section{DISCUSSION}

There have been a number of studies on the mortality rates of pelagic copepod eggs and early nauplii (including those by Kiørboe \& Nielsen 1994, Peterson \& Kimmerer 1994, Ohman \& Hirche 2001, Eiane et al. 2002, Ohman et al. 2002, Eiane \& Ohman 2004). However,

\section{Temperature}

Mortality rates (Lowess-smoothed) were regressed against temperature (Fig. 12). In all cases, except for eggs, there was a strong statistically significant relationship ( $p<0.002)$, with mortality rates increasing with increasing temperature. For eggs, the relationship was not statistically significant $(p>0.05)$. For those cases where a statistically significant relationship was found, the variability in mortality explained by temperature was always higher than $16 \%$, and for NI-NII, $\mathrm{CV}$-adult male and CV-adult female it was greater than $59 \%$. The slopes of the statistically significant relationships ranged from 0.067 to 0.126 , and were broadly similar to those predicted across species by the model of Hirst \& Kiørboe (2002) at 0.073. However, the

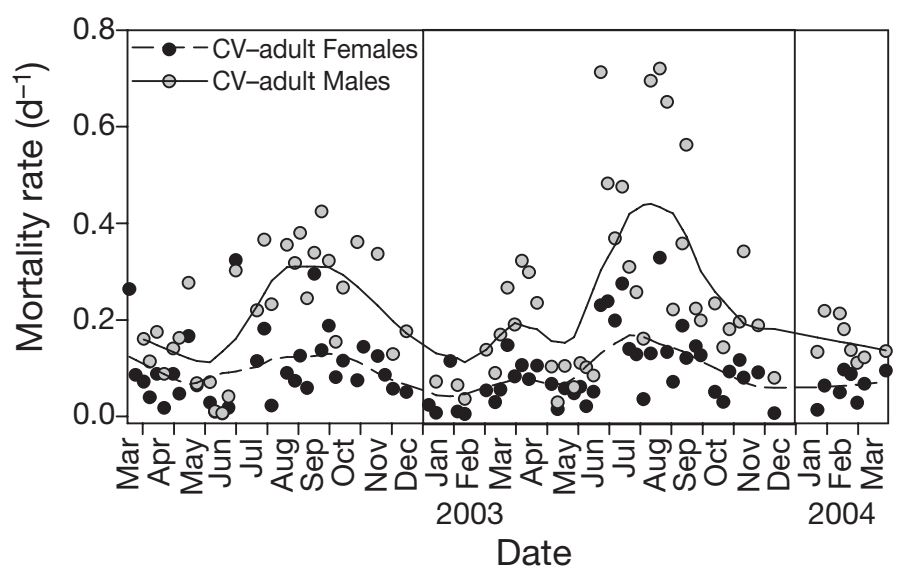

Fig. 8. Calanus helgolandicus. Mortality rates of CV-adult female and $\mathrm{CV}$-adult male through the $2 \mathrm{yr}$ sampling period. Mortality derived from the vertical life-table approach and assuming stage durations were the same as those predicted from the laboratory study. Each point represents solution for an individual date; lines indicate Lowess-fit through all data points few studies on mortality extend beyond a few weeks duration. In our study, mortality rates were estimated on a near-weekly basis over a 2 yr period. This allowed us to compare mortality rates seasonally, and between the 2 years studied. We examined seasonal patterns specifically in the mortality rates of eggs, egg-NI, and CV-adult, because these stages span the whole size/stage range, with the eggs and NI stages being especially critical to continued recruitment.

Our results show that mortality during the egg stage and egg-NI stage pair was on average much greater than in any of the later stage pairs (Figs. 5 \& 6). High rates of mortality in these earliest stages have commonly been observed in many other broadcastspawning copepods (Ianora \& Poulet 1993, Kiørboe \& Sabatini 1994, Peterson \& Kimmerer 1994, Liang \& Uye 1997, see review of Hirst \& Kiørboe 2002). The congeneric species Calanus finmarchicus showed highest mortality rates in eggs, and much lower rates in NI-NII and beyond at Weathership $\mathrm{M}$ in the Norwegian Sea (Ohman et al. 2004). Ohman et al. (2002) measured rates at a coarser stage resolution, but observed highest rates of mortality in C. finmarchicus across the egg-NII stages on Georges Bank. Average rates of mortality for all stage pairs over the course of the present study are given in Fig. 5. The highest values were for egg-NI stages, with an average mortality rate (Method A) of $1.3 \mathrm{~d}^{-1}(95 \% \mathrm{CI}, \pm 0.307)$. A small rise in nauplii mortality rates occurred for the NV-NVI stage pair compared to NIII-NIV and NVI-NV. A similar peak in this mid-life stage was also observed by Ohman et al. (2002) for the Georges Bank population of C. finmarchicus, although it is not evident in this species in other locations (see Ohman et al. 2004). It is worth noting that we computed variance from a series of mortality estimates (i.e. based on single estimates spaced approximately $1 \mathrm{wk}$ apart) without including 


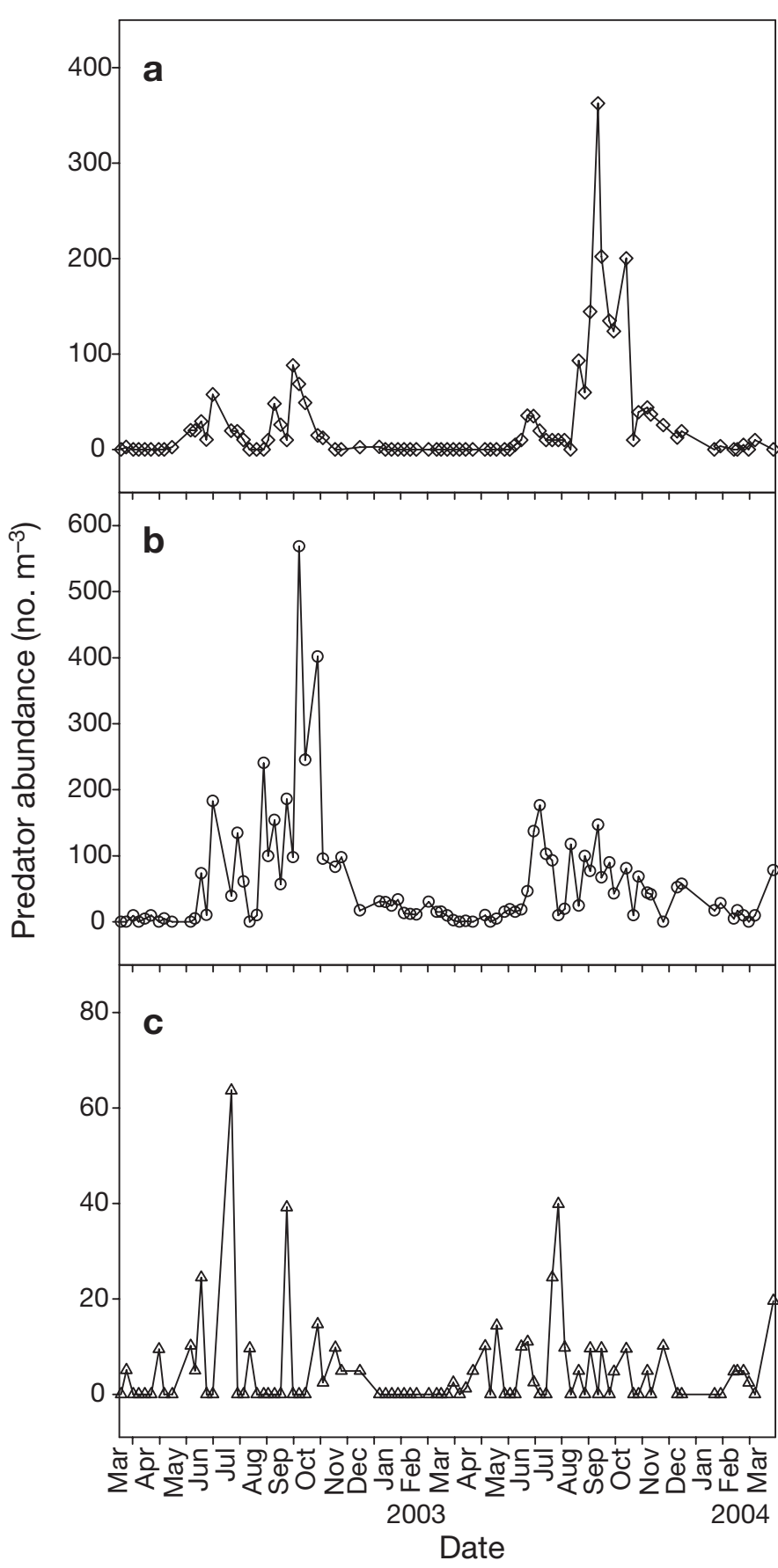

Fig. 9. Abundance of main invertebrate predators at Stn L4 in the $200 \mu \mathrm{m}$ mesh net samples. (a) Siphonophores, (b) chaetognaths and (c) hydromedusae

sampling variance (at each point in time). This procedure differs from that used in some other methods (such as the PSM) where information about sampling variance at each point estimate is included. This is one of the reasons why error terms tend to be larger from PSM estimates than in the present study where VLT has been used.

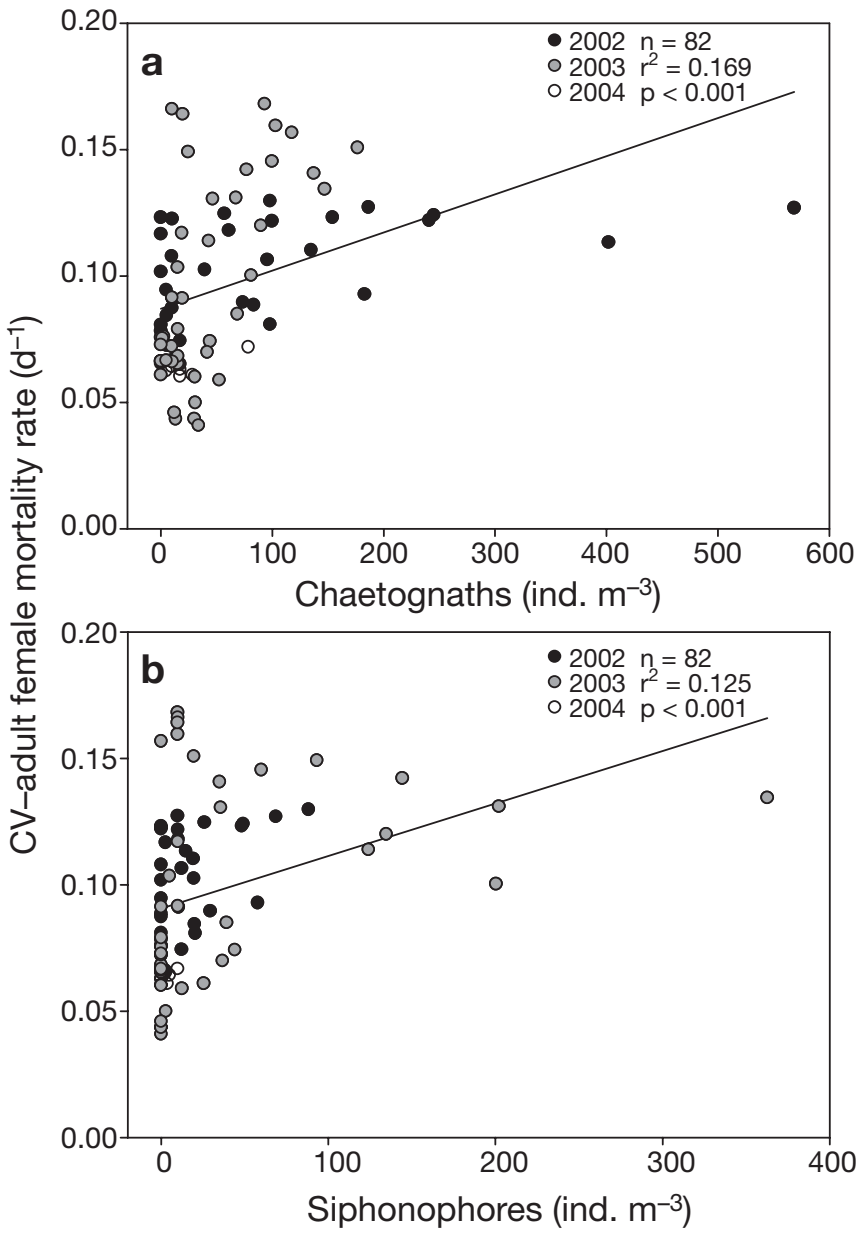

Fig. 10. Calanus helgolandicus. Mortality of CV-adult female stage pair as a function of abundance of (a) chaetognaths and (b) siphonophores. All mortality values are Lowess-smoothed values from the same day as that on which predator abundances were determined. Values for hydromedusae not shown as the regression was not statistically significant $(p>0.05)$

Seasonal egg mortality rates peaked (Lowesssmoothed with f-value set at 0.2) at $8.2 \mathrm{~d}^{-1}$ (Fig. 6a); this is higher but similar to the highest rate of $6.5 \mathrm{~d}^{-1}$ found for the eggs of Calanus finmarchicus in April at Weathership $M$ in the Norwegian Sea (Ohman \& Hirche 2001). Rates of egg mortality that exceed $100 \mathrm{~d}^{-1}$ were reported by Peterson \& Kimmerer (1994), but these appeared not to have been averaged, as is advisable when using a vertical approach, and their magnitude has been questioned on other grounds (Dam \& Tang 2001). Usually less than $10 \%$ of individuals survived through the egg-NI stage pair (Fig. 7), although there were periods in which survival did exceed this value, for example through most of February to June, and also August and September. The mean proportion surviving (Method B) through the egg-NI over the $2 \mathrm{yr}$ of sampling was merely 0.09 

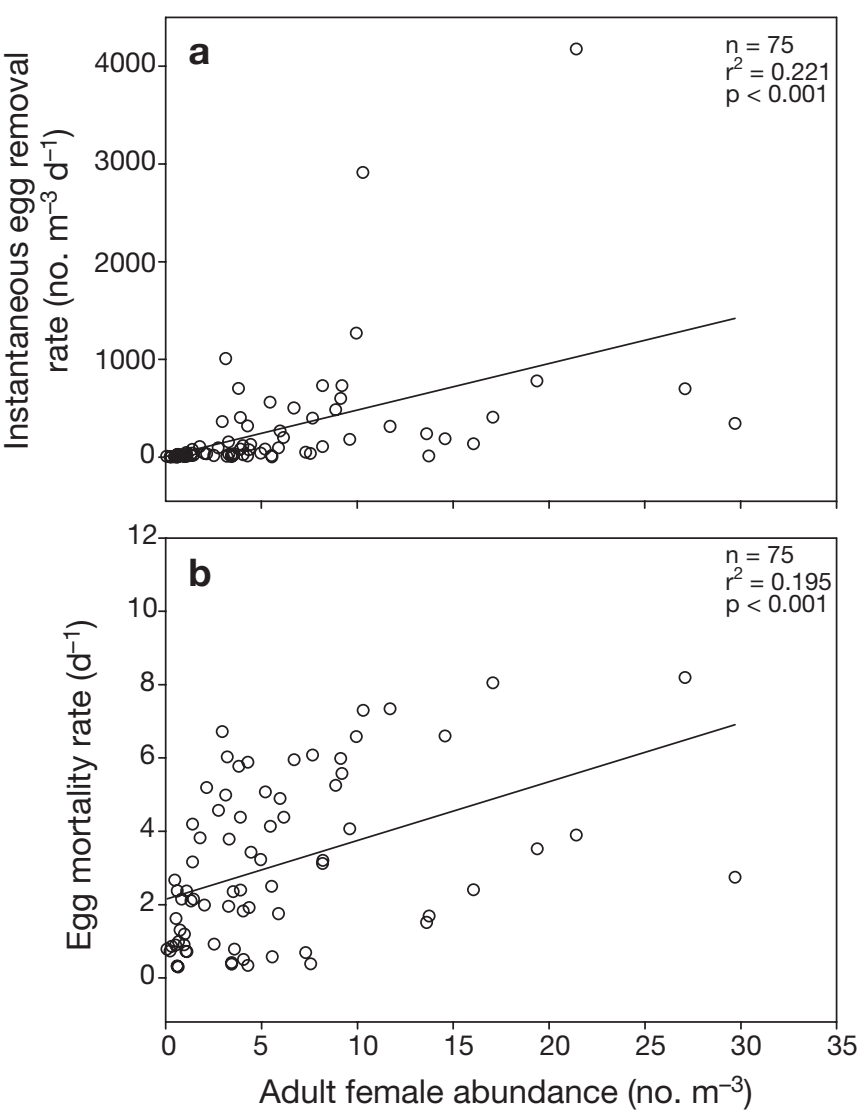

Fig. 11. Calanus helgolandicus. (a) Instantaneous egg removal rates $\left(I_{\mathrm{E}}\right)$ versus abundance of adult females. Relationship described by $I_{\mathrm{E}}=3.37+[47.67 \times$ adult female abundance $\left(\right.$ no. $\left.\mathrm{m}^{-3}\right)$ ]. (b) Mortality of eggs versus abundance of adult females; relationship described by egg mortality rate $=2.142$ $+\left[0.160 \times\right.$ adult female abundance $\left(\right.$ no. $\left.\left.\mathrm{m}^{-3}\right)\right]$. All mortality values are Lowess-smoothed values from same day as that on which adult abundances were determined

(i.e. $9 \%$ ). The proportion that successfully passed through egg-NI varied dramatically over the course of just a few weeks, and over the entire study it varied by more than 5 orders of magnitude. In the same period, the proportion of surviving NV-NVI varied by only $\sim 3$ orders of magnitude, and egg production rates for the population $\mathrm{m}^{-3}$ only rarely exceeded a 3 orders of magnitude range. Although egg production rates are an index of 'potential recruitment', clearly the number of individuals reaching NII (for example) can be poorly related to population egg production rates; hence, the seasonal pattern of these 2 measurements (egg production and NII abundance) can diverge dramatically. Presumably the two may be very poorly related spatially as well as temporally. We highlight this as a warning to others of the limited information that fecundity can provide with respect to actual recruitment to older stages. The seasonal pattern (as percentage surviving through the egg-NI stage pair) is not simply

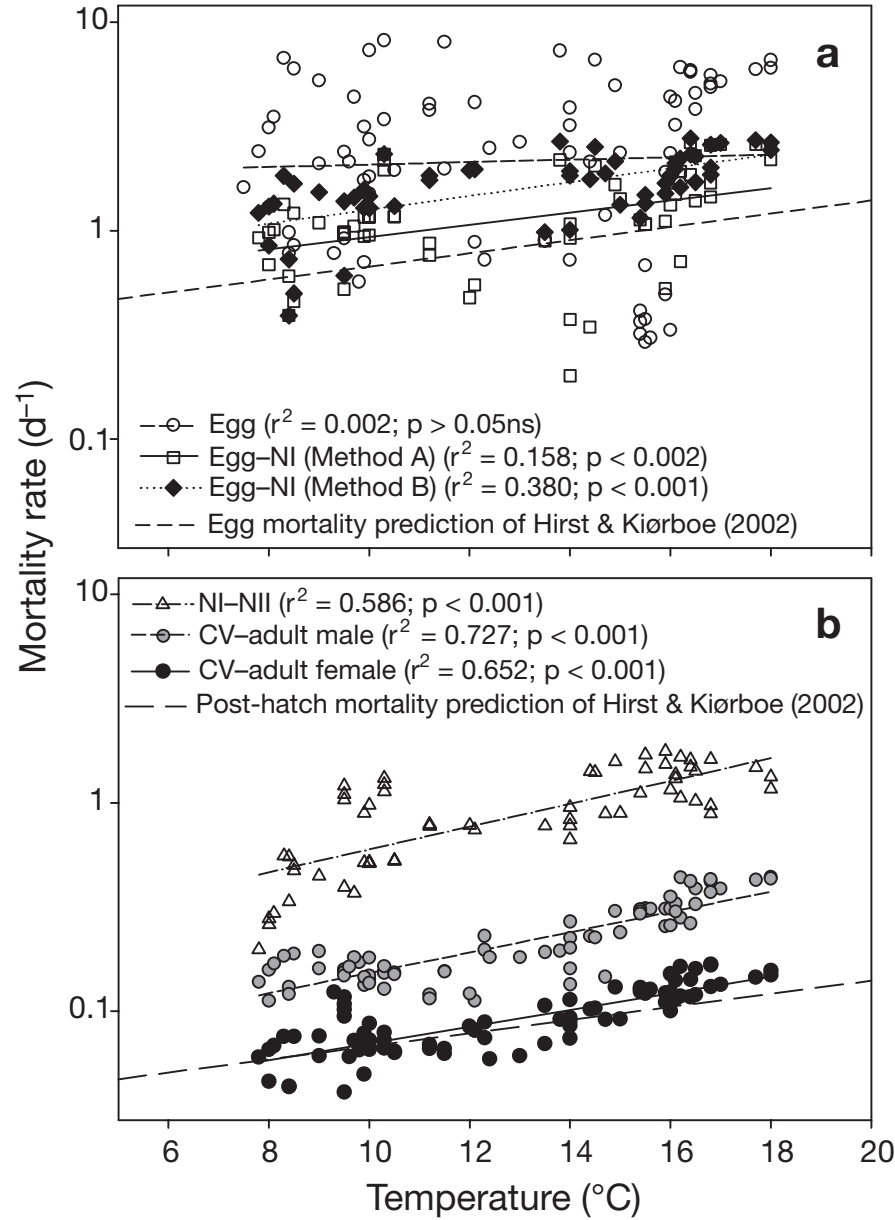

Fig. 12. Calanus helgolandicus. Mortality rates versus surface water temperature. (a) Eggs, egg-NI (Method A) and egg-NI (Method B); (b) NI-NII, CV-adult male and CV-adult female. Values for $\mathrm{CV}$-adult assume that $\mathrm{CV}$ duration is twice that in the laboratory. Relationships are $\log _{\mathrm{e}}$ egg-NI mortality $\left(\mathrm{d}^{-1}\right)=\left(0.067 \times T\left[{ }^{\circ} \mathrm{C}\right]\right)-$ 0.749; $\log _{\mathrm{e}}$ NI-NII mortality $\left(\mathrm{d}^{-1}\right)=\left(0.076 \times T\left[{ }^{\circ} \mathrm{C}\right]\right)-0.531 ; \log _{\mathrm{e}}$ NII-NIII mortality $\left(\mathrm{d}^{-1}\right)=\left(0.126 \times T\left[{ }^{\circ} \mathrm{C}\right]\right)-1.776 ; \log _{\mathrm{e}} \mathrm{CV}$-adult male mortality $\left(\mathrm{d}^{-1}\right)=\left(0.112 \times T\left[{ }^{\circ} \mathrm{C}\right]\right)-3.001 ; \log _{\mathrm{e}} \mathrm{CV}$-adult-female mortality $\left(\mathrm{d}^{-1}\right)=\left(0.092 \times T\left[{ }^{\circ} \mathrm{C}\right]\right)-3.581$. Temperature-dependent predictions of Hirst \& Kiørboe (2002) for copepod eggs and posthatch individuals are shown for comparison. All mortality values are Lowess-smoothed values from same day as that on which unsmoothed mortality values were determined. In all cases except for eggs (ns: not significant) relationships were statistically significant $(\mathrm{p}<0.002)$

proportional to the inverse of the mortality rate of this stage pair. This is because the former considers both mortality rate $\left(\mathrm{d}^{-1}\right)$ and the development time (in days) of these stages; equal rates of mortality can therefore give lower percentage survival when development times are longer (as mortality has more time to occur). This is why the proportion surviving egg-NI was lower in May than in August and September; the 2 periods had similar mortality rates $\left(\mathrm{d}^{-1}\right)$, but development times were much longer during the earlier colder period. 
Calanus finmarchicus eggs sink at a rate of $\sim 0.027 \mathrm{~cm} \mathrm{~s}^{-1}$ (Knutsen et al. 2001), equivalent to $23.3 \mathrm{~m} \mathrm{~d}^{-1}$. Assuming that $C$. helgolandicus eggs sink at a similar rate in the $50 \mathrm{~m}$ deep water column and ignoring turbulence, they would sink from the surface to the bottom in $\sim 2.1 \mathrm{~d}$. The egg hatching time (time to $50 \%$ hatching) is $2.3 \mathrm{~d}$ at $7.5^{\circ} \mathrm{C}$ (coldest winter temperature) and $0.85 \mathrm{~d}$ at $18.0^{\circ} \mathrm{C}$ (warmest summer temperature). A significant proportion of the eggs laid potentially might reach the sediment, and benthic predation might account in part for the high rates of egg mortality at Stn L4. Turbulent mixing is strong at this station, as shown by the lack of thermal stratification throughout most of the year; such mixing may act to reduce the number of eggs reaching the bottom by passive sinking, alternatively it may increase contact of eggs with benthic predators as they mix close the bottom.

The timing and magnitude of peak mortality in the CV-adult stage pair varied between years (Fig. 8). In 2002 mortality peaked in September to October, whereas in 2003 the peak was earlier, in July to August. Mortality rates of these oldest stages were also higher in 2003 than in the previous year. There was an apparent shift in the numerical dominance of the main predators between years, with chaetognaths reaching higher densities in 2002 than in 2003, while siphonophores were more numerous in 2003 than in 2002. The patterns in $\mathrm{CV}$-adult female and male mortality rates (Fig. 8) were similar to total rates in this stage pair. Again peak values occurred 1 to 2 mo earlier in the summer of 2003 than in 2002. The higher mortality in adult males in comparison to females observed in this study has also been observed elsewhere. On Georges Bank, Ohman et al. (2002) found that CV-adult male Calanus finmarchicus had mortality rates $\left(0.09 \mathrm{~d}^{-1}\right)$ nearly twice those of CV-adult females $\left(0.05 \mathrm{~d}^{-1}\right)$. In this study, we found C. helgolandicus to have mortality rates for $\mathrm{CV}$-adult male $\left(0.23 \mathrm{~d}^{-1}\right)$ that were 2 times greater than those of the CV-adult female $\left(0.10 \mathrm{~d}^{-1}\right)$. The sex ratio in adults is often biased towards females in copepods (Hirst \& Kiørboe 2002), which is thought to result from differences in mortality rates between the 2 sexes. Males often have reduced mouthparts and reduced feeding, they may also exhibit different behaviour and increased predation risk over females. We have demonstrated that both the magnitude and seasonal pattern in mortality rates differ between egg-nauplii and CV-adult. This result was generally expected, however, since CVs and adults are much larger than, and display different behaviour to, eggs and NI. Predators are sensitive to prey size and swimming abilities, and we would expect the types of predators and their impacts on mortality to differ dramatically between the 2 age groups (eggs-nauplii and late copepodites) and through time.
Stage durations from experiments on copepods collected from different locations with different acclimation temperatures are often used to determine mortality (e.g. Ohman \& Hirche 2001, Eiane et al. 2002, Eiane \& Ohman 2004), and even data from individuals of the same genus, but different species are used (e.g. Eiane et al. 2002, Ohman et al. 2002, Eiane \& Ohman 2004). Like others, we have used rates determined from individuals collected at a single time of the year and then subjected to a range of temperatures in the laboratory. However, as animals can acclimate to temperature, those taken from different field temperatures might, in the laboratory, show different development times as a function of temperature. Even eggs laid by females accustomed to different temperatures can show differences in development times (e.g. Abou Debs \& Nival 1983). In addition, most studies on development times are conducted in the laboratory under conditions of high algal concentrations, and thus the results may not reflect the field situation. These issues of temperature acclimation and degree of food limitation (in feeding stages) would result in errors in our estimates of mortality when stage durations are not predicted correctly; other studies using similar methods might also encounter these problems. We explored the issue of food limitation by predicting rates of mortality when stage durations in feeding stages were twice those predicted from data in Table 1. These results are presented as annual averages in Fig. 5. Average mortality rates were higher across NII-NIII because, while the duration of NIII is increased by food limitation, the NII stage is non-feeding, and thus we left its duration unaltered. Beyond this stage pair, mortality rates were lower when stage durations were longer. However, the general pattern was very similar, with peaks across the NV-NVI stages and CV-adult. Of course this pattern would not hold true if the degree of food limitation changed with stage. The stage duration-corrected field abundances for NIII were higher than could be accounted for by NII (Fig. 3), and we suggest that this strongly indicates that development times for the feeding nauplii are longer in the field than under approximately food-unlimited conditions in the laboratory. We discount the possibility of this being a net sampling-efficiency effect, as both stages have similar body dimensions and we would not expect a $50 \mu \mathrm{m}$ mesh net to sample them with very different efficiencies.

For mortality estimates, water bottles have been recommended for sampling in preference to pump samples (Ohman et al. 2002). Our net sampling approach has considerable practical advantages; fully depthintegrated samples could be take at this station without the problem of attempting to design a sampling profile that would adequately sample all stages at all depths 
through time. We do stress that the 50 and $200 \mu \mathrm{m}$ nets sampled copepodite stages with very different efficiencies. On average, Copepodite Stage CI was more numerous in the finer-mesh net than in the coarse net, and the older larger copepodite stages were dramatically undersampled by the $50 \mu \mathrm{m}$ net.

\section{Mortality in relation to predator numbers and temperature}

The main pelagic predators at Stn L4 include both vertebrates (fishes and fish larvae) and invertebrates. Although fish larvae were enumerated in samples, we did not believe these were collected quantitatively because of the sampling method used (WP-2, $200 \mu \mathrm{m}$ mesh, vertical hauls). We cannot discount that fish larvae are an important predator on Calanus helgolandicus during some parts of the year, but unfortunately we were unable to estimate their feeding demand. We consider that, in general, the other main planktonic invertebrate predators would have been collected quantitatively by our sampling methods. The main potential predators of late-stage copepodites were chaetognaths, siphonophores and hydromedusae. Chaetognaths were dominated numerically by Sagitta setosa while Muggiaea atlantica was the most numerous siphonophore. Mortality rates of C. helgolandicus CV-adult were significantly related to the abundance of predators in the case of chaetognaths $(p<0.001)$ and siphonophores $(p<$ 0.001) (Fig. 10a and b, respectively), but not hydromedusae ( $p>0.05)$. However, the abundance of chaetognaths and siphonophores accounted for only 17 and $12 \%$ of the variability in mortality, respectively. Backwards stepwise regression analysis resulted in a marked improvement in the variability which could be explained, with a $\mathrm{r}^{2}=0.276(\mathrm{p}<0.001)$ (mortality of $\mathrm{CV}$-adult female $\mathrm{d}^{-1}=0.0813+[0.000157 \times$ siphonophore abundance $\left(\right.$ no. $\left.\left.\mathrm{m}^{-3}\right)\right]+[0.000123 \times$ chaetognath abundance $\left(\right.$ no. $\left.\left.\mathrm{m}^{-3}\right)\right]+[0.000652 \times$ hydromeduase abundance $\left(\right.$ no. $\left.\left.\mathrm{m}^{-3}\right)\right]$ ). Ultimately we are therefore unable to account for $\sim 70 \%$ of the variability in mortality of late-stage C. helgolandicus (CV-adult female) at Stn L4 using abundance of the dominant invertebrate predators. The size of predators was not measured here; as predation capability is strongly size-dependent we suggest that more of the variability in $\mathrm{CV}$-adult mortality rates might have been explained using predator size and abundance measurements and this may be a productive avenue to explore in the future. Feeding rates of $M$. atlantica on copepods can range from 1.2 to 10.5 prey d $^{-1}$ (Purcell 1982). S. setosa and S. elegans consumption rates on copepods have been estimated at 0.75 to 3.6 prey d $^{-1}$ (Mironov 1960, Øresland 1987, Alvarez-Cadena 1993). With densities of siphonophores and chaetognaths often exceeding $>100$ ind. $\mathrm{m}^{-3}$ through much of June to October, they could be potentially removing $\sim 100$ to 1000 prey items $\mathrm{m}^{-3} \mathrm{~d}^{-1}$. This removal would of course be spread among the entire copepod community and over many stages of C. helgolandicus. By multiplying CV-adult (female and male) mortality rates by their abundance, we obtained instantaneous removal rates; the resulting values almost never exceeded a removal rate for the stage pair of 5 ind. $\mathrm{m}^{-3} \mathrm{~d}^{-1}$, and were typically $<2$ ind. $\mathrm{m}^{-3} \mathrm{~d}^{-1}$. From these simple calculations, these 2 predators could potentially account for $C$. helgolandicus $\mathrm{CV}$-adult removal rates through much of June to October.

Through January to May, mortality rates of CV-adult can be variable and relatively high (Fig. 8). However, mortality rates alone can give a distorted picture as to the actual removal of animals. Over the January to May period abundances were low, and rates of removal rarely exceeded $6 \mathrm{CV}$-adult individuals $\mathrm{m}^{-3} \mathrm{~d}^{-1}$. Sometimes it was possible to attribute the removal rates over this period to predator consumption potential, sometimes not; predator abundances were low and their appearance sporadic. Better identification of the causes of mortality throughout this period could prove important, especially if this in turn ultimately controls winter abundance. It has been demonstrated from Continuous Plankton Recorder records that the abundance of planktonic copepods in winter can be critical in determining their abundance later in the year (Colebrook 1985). More field work is needed in order to identify whether winter mortality has a physiological cause, for example starvation, or is the result of direct predation (benthic or pelagic) or disease.

Like many other copepod species, Calanus helgolandicus can feed cannibalistically (Bonnet et al. 2004). As cannibalism is believed to be common in natural populations, many studies have compared the mortality rates of copepod eggs and early nauplii with the abundance of adults (e.g. Peterson \& Kimmerer 1994, Ohman \& Hirche 2001, Ohman et al. 2002 all observed statistically significant positive relationships). When we compare egg mortality rates and instantaneous egg removal rates (the product of mortality and egg density) with the abundance of adult female $C$. helgolandicus, we also observe significant positive linear regressions. Instantaneous egg removal rates $\left(I_{\mathrm{E}}\right.$, no. of eggs removed $\mathrm{m}^{-3} \mathrm{~d}^{-1}$ ) are well correlated with the abundance of females, and described by the relationship $I_{\mathrm{E}}=3.37+\left[47.67 \times\right.$ adult female abundance $\left(\right.$ no. $\left.\left.\mathrm{m}^{-3}\right)\right]$ $\left(\mathrm{r}^{2}=0.221, \mathrm{n}=75, \mathrm{p}<0.001\right)$. If this slope were purely a consequence of cannibalism it would suggest that each female would consume $\sim 48$ eggs $\mathrm{d}^{-1}$. Is this reasonable? Bonnet et al. (2004) found that adult female C. helgolandicus ingestion approaches 20 eggs 
ind. ${ }^{-1} \mathrm{~d}^{-1}$ when egg concentrations are of the order 60000 to 80000 eggs $\mathrm{m}^{-3}$; however, average egg abundance integrated over the water column rarely exceeded 300 eggs $\mathrm{m}^{-3}$ in the field in our study. Of course egg and adult distribution will be patchy, and densities of both are likely to be much higher in the surface waters; nonetheless there is a discrepancy between egg density and the potential clearance rate of adult females, such that cannibalism appears unable to account for the mortality rates observed. Allowing for the presence of younger stages would of course increase the possible predation pressure; however, regressions that included younger copepodites together with the adults produced less significant relationships in all cases tested. Our results show some similarity to those for Temora longicornis in Long Island Sound reported by Peterson \& Kimmerer (1994), who found that egg mortality rates were significantly related to the clearance rate of late stages. However, the mortality rates in their study were far too high to be accounted for by cannibalism. In our study, there was evidence of density-dependence in the mortality rates of C. helgolandicus eggs, with higher mortality rates at higher adult densities (Fig. 11b; p < 0.001), but as already indicated this density-dependence is unlikely to have been attributable to cannibalism alone. Ohman \& Hirche (2001) and Ohman et al. (2002) found that the mortality rates of $C$. finmarchicus eggs and eggs-NII, respectively, were density-dependent; however, adult abundances were much greater in their studies than those observed here.

We regressed mortality rates of non-feeding stages (eggs, egg-NI, NI-NII) and the CV-adult male and CV-adult female stages against surface temperature. We have to be cautious with such an analysis because the 2 axes are not independent of each other, and consequently errors in development time prediction could generate false relationships between mortality and temperature. In all cases except for eggs, there were strongly significant correlations (Fig. 12), with mortality increasing with increasing temperature. In the case of $\mathrm{CV}$-adult male and $\mathrm{CV}$-adult female the surface water temperature explained 73 and $65 \%$ of the variability in mortality, respectively (Fig. 12b); for egg-NI this was $>15 \%$. We suggest that temperature may be a good proxy of other factors such as the total rate of consumption by predators, since both feeding rates and biomass of predators may increase at higher temperatures. Hirst \& Kiørboe (2002) demonstrated that longevity of adult copepods is significantly related to temperature even when they are free from predation (i.e. in laboratory investigations without predators - see their Fig. 7). Metabolism increases with increasing temperature, and there is an acknowledged thermal cost on animals. It is interesting that non-feeding and feeding stages seem to show similar temperature-dependence of mortality, even though the latter stages may be able to meet higher respiration costs through increased feeding. Mortality rates of CV-adult females in the field showed a relationship to temperature of $\log _{\mathrm{e}} \beta_{\mathrm{CV} \text {-adult female }}$ $=0.092 T-3.581$, which compares well with the model prediction of $\log _{e} \beta=0.0725 T-3.157$, where $T$ is temperature $\left({ }^{\circ} \mathrm{C}\right)$ and $\beta$ is mortality $\left(\mathrm{d}^{-1}\right)$. Implicit in the model constructed by Hirst \& Kiørboe (2002) is their prediction that rates are representative of adult females (rather than adult males); indeed the CV-male mortality rates are higher than the model. Mortality rates of eggs and non-feeding nauplii stages at Stn L4 were greater than the model predictions (Fig. 12a), although the slopes (i.e. temperature-dependence), which ranged from 0.067 to 0.126 , compare well with Hirst \& Kiørboe's (2002) prediction of 0.0725 .

\section{Comparing mortality rates between methods}

The mortality rates of egg-NI obtained by Methods $\mathrm{A}$ and $\mathrm{B}$ are compared in Fig. 13. The 2 methods gave similar values; the data generally fell about the 1:1 line, and around half fell within 50 to $150 \%$ of the value obtained by the other method (dashed lines in Fig. 13). However, on average Method A values were lower. Method A, which involves the enumeration of eggs in the water column, gave lower rates of mortality

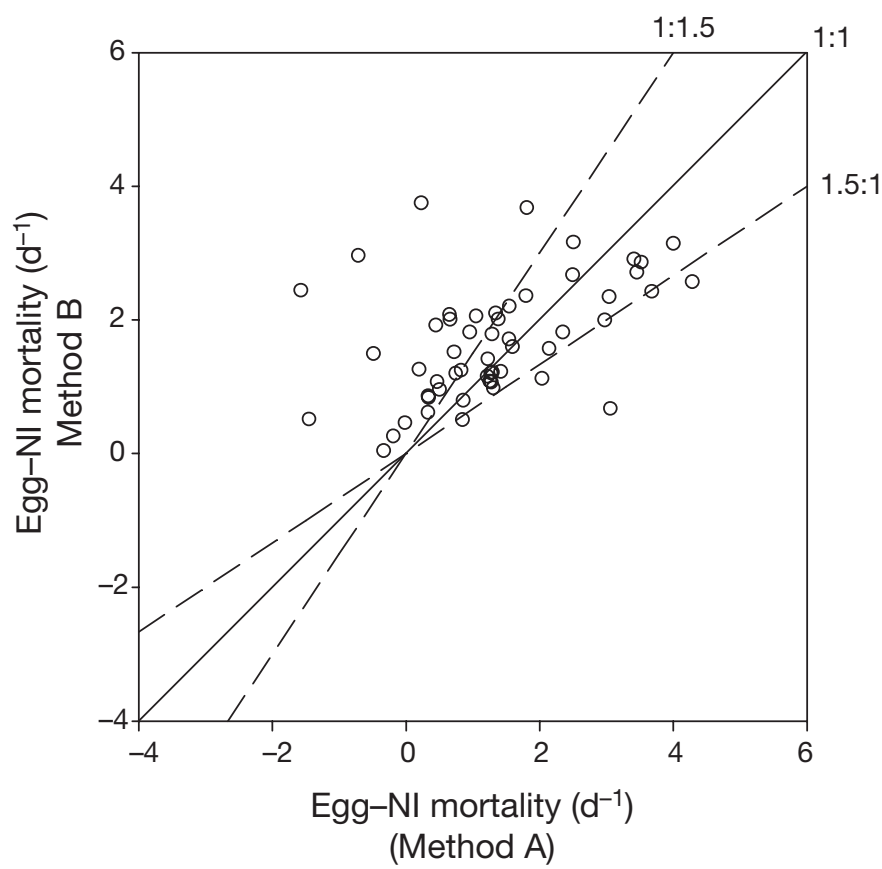

Fig. 13. Calanus helgolandicus. Egg-NI mortality rates $\left(\mathrm{d}^{-1}\right)$ derived by Methods A and B. Continuous line: 1:1 ratio of Method A to Method $B_{\text {; }}$ dashed lines: 1.5:1 and 1:1.5 ratios. Lowess-smoothed values were not used; instead we used mortality solutions on each sample date 
than Method B, which uses egg production results to estimate egg input possibly because (1) field abundance of eggs sampled with the $50 \mu \mathrm{m}$ net were underestimated relative to NI (this would result in underestimation of mortality by Method A); (2) the abundance of eggs and NI were underestimated in the $50 \mu \mathrm{m}$ net relative to adults in the $200 \mu \mathrm{m}$ net (this would result in overestimation of mortality by Method B); or (3) egg production rates from incubations overestimated fecundity rates in the natural environment (this would result in overestimation of mortality by Method B). These 3 reasons are not mutually exclusive, and the difference would almost certainly vary between different sampling methods used. The presence of nonhatching eggs in the population is another factor which would systematically result in errors by Method A (egg-NI) and the egg mortality method, although it would result in overestimation in Method A compared to Method B. We turn our attention to method corrections for this below.

\section{Considering hatching success in mortality equations}

It is commonly observed that a variable proportion of eggs produced by copepods do not hatch. However, both Eq. (2) (Method A) applied across the egg-NI stage pair, and Eq. (5) applied to eggs, fail to consider this. Both equations consider that eggs have a hatching time that is consistent with the Bêlehràdek prediction, yet this is clearly not the case when non-viable eggs are laid. These equations have been frequently used and we feel it is timely that we point out this error and present a method to correct for it. We re-write the equations to consider 2 'populations' of eggs, one which hatches at a rate according to the Bêlehràdek predictions, and one comprising non-hatching eggs. We then compare results using both the unmodified and newly modified formulae for Stn L4.

\section{Egg mortality rates}

Eq. (5) has been used to derive egg mortality rates; however, it fails to consider those eggs that will not hatch. It can be re-written to allow for the nonhatching eggs as:

or

$$
\begin{gathered}
N_{\text {egg }}=\pi_{1} N_{\text {adult_female }} F\left[1-\exp \left(-\beta_{\mathrm{c}} D_{1}\right)\right] / \\
\beta_{\mathrm{c}}+\pi_{2} N_{\text {adult_female }} F\left[1-\exp \left(-\beta D_{2}\right)\right] / \beta_{\mathrm{c}}
\end{gathered}
$$

$$
\begin{aligned}
& N_{\text {egg }} \beta_{\mathrm{c}} / N_{\text {adult_female }} F=\pi_{1}\left[1-\exp \left(-\beta_{\mathrm{c}} D_{1}\right)\right] \\
& +\pi_{2}\left[1-\exp \left(-\beta_{\mathrm{c}} D_{2}\right)\right]
\end{aligned}
$$

where $\beta_{\mathrm{c}}$ is the egg mortality rate $\left(\mathrm{d}^{-1}\right)$ allowing for 2 populations of eggs, $D_{\mathrm{k}}$ is the hatching time for eggs of
Type $\mathrm{k}(\mathrm{d})$, and $\pi_{\mathrm{k}}$ is the proportion of eggs laid of Type $\mathrm{k}$ (we set Type 1 as the actively hatching population and Type 2 as the non-hatching eggs - the relative proportion of these comes from the egg hatching success experiments). Eqs. (8) \& (9) assume that the mortality rate of the 2 populations of eggs is equal, and only mortality removes the non-hatching eggs. For given values of $N_{\text {egg }}, N_{\text {adult_female }}, F_{1} \pi_{1}, \pi_{2}, D_{1}$ and $D_{2}$, the value of $\beta_{\mathrm{c}}$ is thus obtained using an iterative method. We solve for $\beta_{\mathrm{c}}$ (egg mortality) assuming $D_{2}$ (the time to hatching for those eggs incapable of hatching) to be an extremely large number. Using the data from Stn L4 we are able to compare results from Eq. (9), which considers the bias introduced by production of non-hatching eggs, and Eq. (5), which fails to account for such eggs. We find that the 2 methods produce very similar results (Fig. 14) at higher mortality rates (i.e. $>2 \mathrm{~d}^{-1}$ ), but diverge more at lower mortality rates. At low mortality rates the 2 measurements diverge more because of the rising proportion of nonhatching eggs in the water column. Note how Eq. (9) (corrected for the presence of non-hatching eggs) gives mortality estimates that are never lower than those from Eq. (5). This is because egg abundance is inflated as a result of the presence (and longer residence) of non-hatching eggs. Use of the uncorrected equation for egg mortality may have led others in the

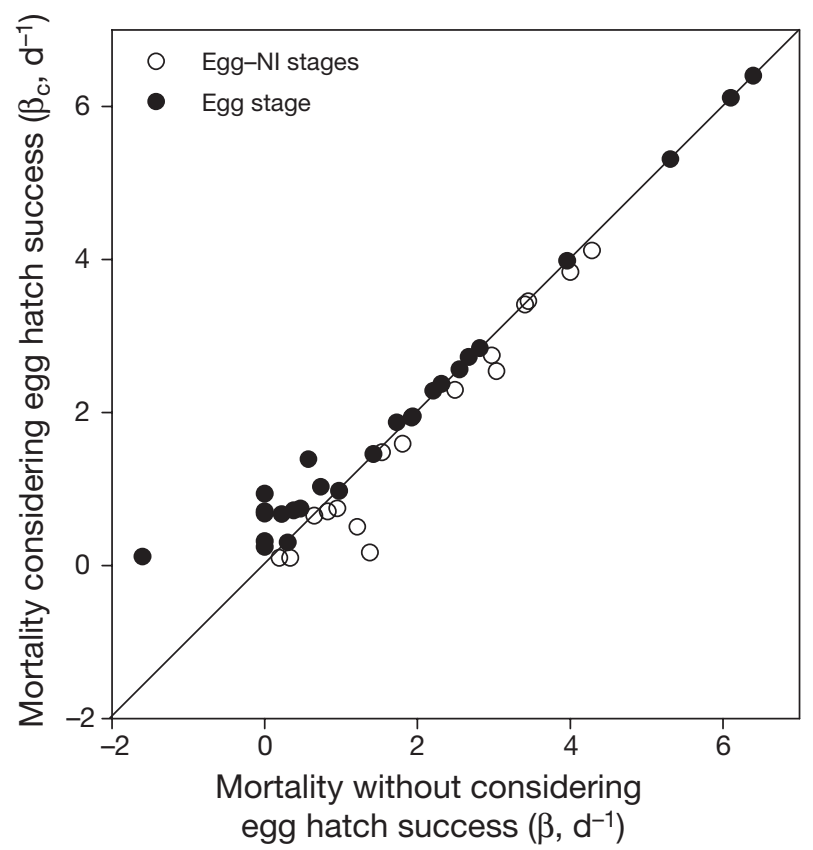

Fig. 14. Calanus helgolandicus. Comparison of egg mortality rates obtained from Stn L4 data set using equations that consider proportion of eggs that do not hatch (Eq. 9 for eggs, Eq. 10 for egg-NI), with rates from equations that fail to consider non-hatching eggs (Eq. 2 for eggs and Eq. 5 [Method A] for egg-NI). Lowess-smoothed values were not used; instead we used mortality solutions on each sample date 
past to underestimate the true mortality rate, especially when mortality rates were less than $\sim 2 \mathrm{~d}^{-1}$. The lower the egg hatching success, the greater the deviation in results obtained from the 2 equations. Although mortality rates from Eqs. (9) \& (5) were often similar in this study, this was largely because mortality rates were often $>1 \mathrm{~d}^{-1}$, and hatching success was commonly high. Other investigators need to consider using Eq. (9) if the mortality rates in their study are low and/or egg hatching success is poor.

\section{Egg-NI mortality rate}

Eq. (2) can be re-written for mortality across the egg-NI stage pair, allowing again for the presence of eggs that do not hatch, as:

$$
\begin{aligned}
& N_{\text {egg }} / N_{\mathrm{NI}}=\left\{1-\left[\pi_{1} \exp \left(-\beta_{\mathrm{c}_{-} \text {egg-NI }} D_{1}\right)\right.\right. \\
& \left.\left.+\pi_{2} \exp \left(-\beta_{\mathrm{c}_{-} \text {egg-NI }} D_{2}\right)\right]\right\} /\left\{\left[\pi_{1} \exp \left(-\beta_{\mathrm{c}_{-} \text {egg-NI }} D_{1}\right)\right.\right. \\
& \left.\left.+\pi_{2} \exp \left(-\beta_{\mathrm{c} \_ \text {egg-NI }} D_{2}\right)\right]\left[1-\exp \left(-\beta_{\mathrm{c} \_ \text {egg-NI }} D_{\mathrm{NI}}\right)\right]\right\}
\end{aligned}
$$

where $\beta_{\mathrm{c}_{-} \text {egg-NI }}$ is the mortality $\left(\mathrm{d}^{-1}\right)$ across the egg-NI stage pair allowing for the 2 populations of egg types. In Fig. 14, we compare results from Eqs. (2) \& (10) using the Stn L4 data. We see that the correction leads to a reduction in mortality estimates. Method B (Eq. 4) does not include egg abundance, and hence will not be biased by the non-hatching eggs. Again we urge others to consider using the more complex equation in the future.

Acknowledgements. This work was funded by the NERC Marine Productivity Thematic Programme (Grant Refs. NER/ T/S/1999/00057), and is a contribution to BAS Discovery 2010 and to the Plymouth Marine Laboratory Core Research Programme. We thank Tania Smith for help with the plankton analysis. Mark Ohman kindly gave advice on our initial data set. Ketil Eiane provided insightful comments on our analyses. Finally, we thank Pete Rothery for his invaluable mathematical help in deriving egg mortality equations.

\section{LITERATURE CITED}

Abou Debs C, Nival P (1983) Etude de la ponte et du développement embryonnaire en relation avec la température et la nourriture chez Temora stylifera Dana (Copepoda, Calanoida). J Exp Mar Biol Ecol 72:125-145

Aksnes DL, Ohman MD (1996) A vertical life-table approach to zooplankton mortality estimation. Limnol Oceanogr 41: 1461-1469

Aksnes DL, Miller CB, Ohman MD, Wood SN (1997) Estimation techniques used in studies of copepod population dynamics - a review of underlying assumptions. Sarsia 82:279-296

Alvarez-Cadena JN (1993) Feeding of the chaetognath Sagitta elegans Verrill. Estuar Coast Shelf Sci 36:195-206

Bonnet D, Titelman J, Harris RP (2004) Calanus the cannibal. J Plankton Res 26:937-948
Bunker AJ, Hirst AG (2004) Fecundity of marine planktonic copepods: global rates and patterns in relation to chlorophyll $a$, temperature and body weight. Mar Ecol Prog Ser 269:161-181

Campbell RG, Wagner MM, Teegarden GJ, Boudreau CA, Durbin EG (2001) Growth and development of the copepod Calanus finmarchicus reared in the laboratory. Mar Ecol Prog Ser 221:161-183

Colebrook JM (1985) Continuous plankton records: overwintering and annual fluctuations in the abundance of zooplankton. Mar Biol 84:261-265

Continuous Plankton Recorder Survey Team (2004) Continuous plankton records: plankton atlas of the North Atlantic Ocean (1958-1999). II. Biogeographical charts. Mar Ecol Prog Ser CPR(Suppl):11-75

Corkett CJ (1972) Development rate of copepod eggs of the genus Calanus. J Exp Mar Biol Ecol 10:171-175

Corkett CJ, McLaren IA, Sevigny JM (1986) The rearing of the marine calanoid copepods Calanus finmarchicus (Gunnerus), C. glacialis Jaschnov and C. hyperboreus Krøyer with comments on the equiproportional rule. Syllogeus 58:539-546

Dam HG, Tang KM (2001) Affordable egg mortality: constraining copepod egg mortality with life history traits. J Plankton Res 23:633-640

Eiane K, Ohman MD (2004) Stage-specific mortality of Calanus finmarchicus, Pseudocalanus elongatus and Oithona similis on Fladen Ground, North Sea, during a spring bloom. Mar Ecol Prog Ser 268:183-193

Eiane K, Aksnes DL, Ohman MD, Wood S, Martinussen MD (2002) Stage-specific mortality of Calanus spp. under different predation regimes. Limnol Oceanogr 47:636-645

Hirst AG, Bunker AJ (2003) Growth of marine planktonic copepods: global rates and patterns in relation to chlorophyll $a$, temperature, and body weight. Limnol Oceanogr 48:1988-2010

Hirst AG, Kiørboe T (2002) Mortality of marine planktonic copepods: global rates and patterns. Mar Ecol Prog Ser 230:195-209

Holligan PM, Harbour DS (1977) The vertical distribution and succession of phytoplankton in the Western English Channel in 1975 and 1976. J Mar Biol Assoc UK 57:1075-1093

Holligan PM, Harris RP, Newell RC, Harbour DS, Head RN, Linley EAS, Lucas MT (1984) Vertical distribution and portioning of organic carbon in mixed, frontal and stratified waters of the English Channel. Mar Ecol Prog Ser 14: 111-127

Ianora A, Poulet SA (1993) Egg viability in the copepod Temora stylifera. Limnol Oceanogr 38:1615-1626

Irigoien X, Harris RP (2003) Interannual variability in Calanus helgolandicus dynamics. Fish Oceanogr 12:317-326

Kiørboe T, Nielsen TG (1994) Regulation of zooplankton biomass and production in a temperate, coastal ecosystem. 1. Copepods. Limnol Oceanogr 39:493-507

Kiørboe T, Sabatini M (1994) Reproductive and life cycle strategies in egg-carrying cyclopoids and free-spawning calanoid copepods. J Plankton Res 16:1353-1366

Knutsen T, Melle W, Calise L (2001) Determining the mass density of marine copepods and their eggs with a critical focus on some of the previously used methods. J Plankton Res 23:859-873

Liang D, Uye S (1997) Population dynamics and production of the planktonic copepods in a eutrophic inlet of the Inland Sea of Japan. IV. Pseudodiaptomus marinus, the eggcarrying calanoid. Mar Biol 128:415-421

Mironov GN (1960) The food of plankton predators. 2. Food of Sagitta. Tr Sevastopol Biol Sta 13:78-88 
Mullin MM, Brooks ER (1970) Production of the planktonic copepod Calanus helgolandicus. Bull Scripp Inst Oceanogr 17:89-103

Ohman MD, Hirche HJ (2001) Density-dependent mortality in an oceanic copepod population. Nature 412:638-641

Ohman MD, Runge JA, Durbin EG, Field DB, Niehoff B (2002) On birth and death in the sea. Hydrobiologia 480:55-68

Ohman MD, Eiane K, Durbin EG, Runge JA, Hirche JA (2004) A comparative study of Calanus finmarchicus mortality patterns at five locations in the North Atlantic. ICES J Mar Sci 61:687-697

Øresland V (1987) Feeding of the chaetognaths Sagitta elegans and $S$. setosa at different seasons in the western English Channel. Mar Ecol Prog Ser 39:69-79

Peterson WT, Kimmerer WJ (1994) Processes controlling recruitment of the marine calanoid copepod Temora longicornis in Long Island Sound: egg production, egg mortality, and cohort survival rates. Limnol Oceanogr 39:1594-1605

Pingree RD, Holligan PM, Mardell GT (1978) The effects of vertical stability on phytoplankton distributions in the

Editorial responsibility: Howard Browman (Associate Editorin-Chief), Storebø, Norway summer on the northwest European shelf. Deep-Sea Res 25:1011-1028

Pond D, Harris R, Head R, Harbour D (1996) Environmental and nutritional factors determining seasonal variability in the fecundity and egg viability of Calanus helgolandicus in coastal waters off Plymouth, UK. Mar Ecol Prog Ser 143: 45-63

Purcell JE (1982) Feeding and growth of the siphonophore Muggiaea atlantica (Cunningham 1893). J Exp Mar Biol Ecol 62:39-54

Rey C, Harris R, Irigoien X, Head R, Carlotti F (2001) Influence of algal diet on growth and ingestion of Calanus nauplii. Mar Ecol Prog Ser 216:151-165

Thompson BM (1982) Growth and development of Pseudocalanus elongatus and Calanus sp. in the laboratory. J Mar Biol Assoc UK 62:359-372

Uye SI, Yamaoka T, Fujisawa T (1992) Are tidal fronts good areas for herbivorous copepods? Fish Oceanogr 1, 3:216-226

Wood SN (1994) Obtaining birth and mortality patterns from structured population trajectories. Ecol Monogr 64:23-44

Submitted: June 30, 2006; Accepted: October 25, 2006

Proofs received from author(s): May 28, 2007 